Draft VERSion MARCh 11, 2022

Preprint typeset using $\mathrm{IAT}_{\mathrm{E}} \mathrm{X}$ style emulateapj v. 5/2/11

\title{
THE MASSES OF LOCAL GROUP DWARF SPHEROIDAL GALAXIES: THE DEATH OF THE UNIVERSAL MASS PROFILE
}

\author{
Michelle L. M. Collins ${ }^{1}$, Scott C. Chapman ${ }^{2,3}$, R. M. Rich ${ }^{4}$, Rodrigo A. Ibata ${ }^{5}$, Nicolas F. Martin ${ }^{5,1}$, Michael J. \\ Irwin $^{2}$, Nicholas F. Bate ${ }^{6}$, Geraint F. Lewis ${ }^{6}$, Jorge Peñarrubia ${ }^{7}$, Nobuo Arimoto ${ }^{8,9}$, Caitlin M. Casey ${ }^{10}$, \\ Annette M. N. Ferguson ${ }^{11}$, Andreas Koch ${ }^{12}$, Alan W. McConnachie ${ }^{13}$, Nial Tanvir ${ }^{14}$ \\ Draft version March 11, 2022
}

\begin{abstract}
We investigate the claim that all dwarf spheroidal galaxies (dSphs) reside within halos that share a common, universal mass profile as has been derived for dSphs of the Galaxy. By folding in kinematic information for 25 Andromeda dSphs, more than doubling the previous sample size, we find that a singular mass profile can not be found to fit all the observations well. Further, the best-fit dark matter density profile measured for solely the Milky Way dSphs is marginally discrepant (at just beyond the $1 \sigma$ level) with that of the Andromeda dSphs, where a profile with lower maximum circular velocity, and hence mass, is preferred. The agreement is significantly better when three extreme Andromeda outliers, And XIX, XXI and XXV, all of which have large half-light radii ( dispersions $\left(\sigma_{v}<5 \mathrm{~km} \mathrm{~s}^{-1}\right)$ are omitted from the sample. We argue that the unusual properties of these outliers are likely caused by tidal interactions with the host galaxy.

Subject headings: dark matter — galaxies: dwarf — galaxies: fundamental parameters — galaxies: kinematics and dynamics - Local Group
\end{abstract}

\section{INTRODUCTION}

Within the accepted cosmological paradigm $-\Lambda$ cold dark matter $(\Lambda \mathrm{CDM})$ - approximately $85 \%$ of the matter in the Universe is thought to be dark (Komatsu et al. 2011, Planck Collaboration et al. 2013a b). As such, understanding the nature of this component is of the upmost importance. While the precise properties of this exotic matter are still unknown, various predictions about its behaviour and mass distribution within galaxies have been made by both cosmological and particle physics models.

While it has experienced great success on large scales, this cosmological model has run into some difficulty adequately explaining a number of observations made on

\footnotetext{
${ }^{1}$ Max-Planck-Institut für Astronomie, Königstuhl 17, D69117 Heidelberg, Germany

${ }^{2}$ Institute of Astronomy, Madingley Rise, Cambridge, CB3 OHA ,UK

${ }^{3}$ Dalhousie University Dept. of Physics and Atmospheric Science Coburg Road Halifax, B3H1A6, Canada

${ }^{4}$ Department of Physics and Astronomy, University of California, Los Angeles, CA 90095-1547

${ }^{5}$ Observatoire de Strasbourg,11, rue de l'Université, F-67000, Strasbourg, France

${ }^{6}$ Sydney Institute for Astronomy, School of Physics, A28, University of Sydney, NSW 2006, Australia

${ }^{7}$ Ramón y Cajal Fellow, Instituto de Astrofísica de AndaluciaCSIC, Glorieta de la Astronomía s/n, 18008, Granada, Spain

${ }^{8}$ Subaru Telescope, National Astronomical Observatory of Japan 650 North A'ohoku Place, Hilo, Hawaii 96720, U.S.A.

${ }_{9}^{9}$ Graduate University for Advanced Studies 2-21-1 Osawa, Mitaka, Tokyo 181-8588, Japan

${ }^{10}$ Institute for Astronomy, 2680 Woodlawn Drive Honolulu, HI 96822-1839 USA

${ }^{11}$ Institute for Astronomy, University of Edinburgh, Royal Observatory, Blackford Hill, Edinburgh, EH9 3HJ, UK

12 Zentrum für Astronomie der Universität Heidelberg, Landessternwarte, Königstuhl 12, 69117 Heidelberg, Germany

${ }^{13}$ NRC Herzberg Institute of Astrophysics, 5071 West Saanich Road, British Columbia, Victoria V9E 2E7, Canada

14 Department of Physics \& Astronomy, University of Leicester, University Road, Leicester LE1 7RH, UK
}

smaller scales. In particular, a number of mismatches between observation and theory with regard to the smallest, most dark matter dominated galaxies we are able to observe - the dwarf spheroidals (dSphs) - have been defined. The missing satellite problem has floated around for some time now (Klypin et al. 1999; Moore et al. 1999) and refers to the dearth of observed luminous subhalos around the Milky Way (MW) and Andromeda (M31), compared to the vast number of dark matter subhalos seen within dark matter only simulations. The scope of this problem has somewhat lessened over the years, as the community seems largely satisfied that this can be resolved with future observations and a better understanding of the physics underlying galaxy formation. Firstly, we do not expect all subhalos seen within the simulations to have enough mass to accrete and retain the gas required to efficiently form stars. As such, a lower mass of $V_{\max } \sim 10-15 \mathrm{~km} \mathrm{~s}^{-1}$ is placed on luminous galaxy formation (Peñarrubia et al. 2008b: Koposov et al. 2009). Secondly, our observations are currently incomplete (both areally and in terms of surface brightness). By considering and correcting for the completeness of current surveys of the halos of these galaxies (Tollerud et al. 2008; Koposov et al. 2009, Walsh et al. 2009, Martin et al, in prep.) the number of observed vs. predicted subhalos can be brought into much better agreement.

Another observed and as yet unresolved tension is the ongoing 'cusp-core' debate, which refers to the shape of the dark matter profile of galactic halos as radius tends to zero. With central mass to light ratios of typically $M / L>10 \mathrm{M}_{\odot} / \mathrm{L}_{\odot}$ (e.g Mateo 1998, Walker et al. 2009 Tollerud et al. 2012; Collins et al. 2013), one can treat the stars contained within dSphs as massless tracers of the dark matter potential, and their small scales (halflight radii of $r_{\text {half }} \sim 100-1000 \mathrm{~s} \mathrm{pc}$ ) allow us to probe their mass profiles in the very centers of their halos. This allows us to test the predictions from cosmological dark 
matter only simulations of halo mass profiles; namely that these are steeply cusped (i.e., the density dramatically increases for decreasing radius, Navarro et al. 1997). Increasingly, observations of dwarf spheroidals (and other low surface brightness galaxies) show evidence for constant density cores in the centers of galaxy halos (e.g. de Blok \& Bosma 2002, de Blok et al. 2003; de Blok 2005; Walker \& Peñarrubia 2011; Amorisco \& Evans 2012; Jardel \& Gebhardt 2012). Whether this tension can be resolved by appealing to baryonic processes, such as feedback from star formation or tidal stripping is something that is currently being debated (e.g. Zolotov et al. 2012, Brooks \& Zolotov 2012, Garrison-Kimmel et al. 2013) and a theme we shall return to later on.

Related to the cusp-core problem is the 'too big to fail' (TBTF) problem, which was originally identified by Read et al. (2006) and has received much attention recently from others (e.g. Boylan-Kolchin et al. 2011b, 2012). With the limited kinematic data currently available for $\mathrm{dSph}$ galaxies, it is not possible to accurately measure the slopes of their density profiles in many cases, but from measurements of their central velocity dispersion, $\sigma_{v}$, one can get a good grasp on the central masses, i.e. the mass within the 2-dimensional half-light radius, $r_{\text {half }}$, of these systems (Walker et al. 2009; Wolf et al. 2010), and compare these with those of simulated subhalos. Such an exercise was undertaken by Boylan-Kolchin et al. (2011b) using the Aquarius set of simulations (Springel et al. 2008), and they found that each MW-like Aquarius halo they studied had of order 10 subhalos with central masses that were significantly higher than those of the MW dSphs. This means either the most massive subhalos within MW systems do not necessarily form stars, or that we are missing some crucial physics from these models (either baryonic, or with respect to the properties of dark matter itself) that can explain this discrepancy.

Each of these problems are currently being investigated by observers and theorists alike, with proposed solutions ranging from fiddling with baryonic physics (star formation, feedback, tidal forces etc.) to redefining the cosmological paradigm (e.g. modified Newtonian dynamics, warm dark matter, self interacting dark matter). From the observers point of view, one obvious avenue has been to extend our sample of objects whose kinematics are well measured. Obtaining the necessary kinematic information with which to study the central masses of dSphs (i.e. radial velocities of individual stars within these systems) is exceptionally challenging, meaning the majority of studies thus far have focused on the 20 surrounding our own Galaxy. It has only been within the last decade that telescopes capable of measuring kinematics of extragalactic dSphs have become available. Now, thanks to several recent papers (e.g. Kalirai et al. 2010, Collins et al. 2010, 2011, 2013, Tollerud et al. 2012, 2013), we can add to this sample a further 25 dSphs from the Andromeda system, more than doubling our sample size. Two of these works in particular, Tollerud et al. (2012, henceforth T12) and Collins et al. (2013, henceforth C13) have demonstrated that the majority of the M31 systems have very similar central masses to their MW counterparts, which would imply that the self-same tensions discussed above for the MW also apply to the M31 dSph system. In addition, they highlighted a number of M31 dSphs whose masses appear lower than would be expected when comparing

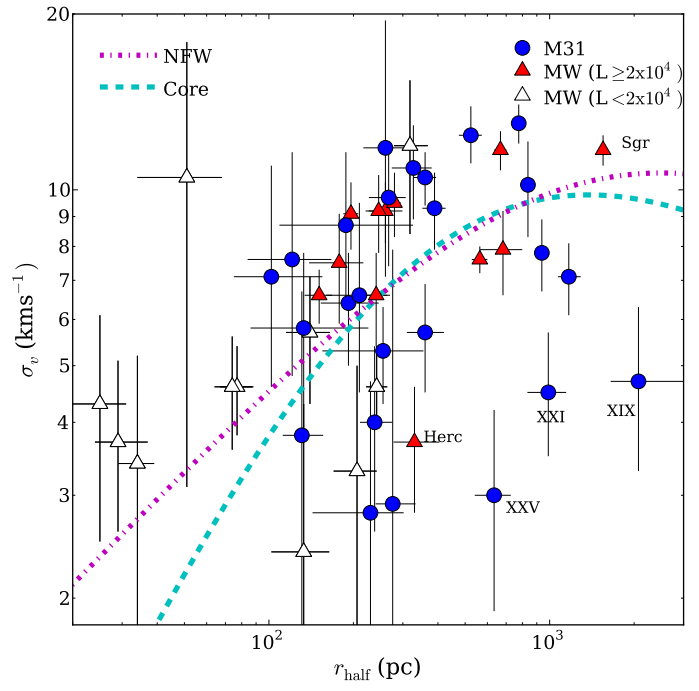

FIG. 1.- Half-light radius vs. velocity dispersion for MW (red triangles) and M31 dSphs (blue circles). Overlaid are best fitting NFW and core mass profiles to these data. Open symbols represent MW dSphs that are too faint to be observed in M31, and hence are excluded from the fits. $\sim 50 \%$ of all observations are inconsistent with these fits, undermining the notion that all dSphs are embedded in halos that follow a universal density profile.

with expectations based on Milky Way dSphs, casting some doubt on the notion that all dSph galaxies are hosted within dark matter halos whose central mass profiles are universal, i.e., behave in a statistically similar way as a function of radius (Mateo 1998, Strigari et al. 2008, Walker et al. 2009, Wolf et al. 2010).

In this work, we revisit the idea of universal mass profile of Walker et al. (2009) for the dSph population by including the M31 objects into the analysis. In $\S[3$ we show that a singular mass profile, be it a Navarro et al. (1997) cusp (NFW) or a constant density core, provides a poor fit to the Local Group dSphs, and instead we advocate a statistical range of best fit mass profiles that track the scatter in mass for a given half-light radius in this population. We then compare these findings with numerical simulations, demonstrating that the mismatches discussed above do not simply go away with a larger sample of systems. We identify a number of unusual systems in M31 whose masses may pose a challenge to our understanding of galaxy formation and evolution in $\S 4$. Finally, in $\S 5$, we go on to discuss how the proposed solutions to these problems stack up to the observations, before we conclude in $\oint 6$.

\section{DATA}

As dSphs are largely dispersion supported systems with little or no evidence of rotation, their velocity dispersions, in combination with their half-light radii, can be utilized to estimate their central masses. For the Milky Way population, we rely on the compilation of kinematic and structural properties formed by Walker et al. (2009), although we exclude the tidally disrupting Sagittarius galaxy ( $\mathrm{Sgr}$ ) from further analysis, as it is currently not in equilibrium. The compilation from Walker et al. (2009) were used within that work to define the universal mass profile for dSph galaxies, which 
we shall discuss further below. Since then, three Galactic dSphs have benefitted from further study of their kinematics; Hercules (Adén et al. 2009), Böotes I (Koposov et al. 2011) and Segue 2 (Kirby et al. 2013). In all cases, the velocity dispersions (and hence, calculated masses) have reduced.

For the Andromeda dSphs, we take our kinematics and structural properties from the final table in $\mathrm{C} 13$, which is a compilation of the best velocity dispersions from that work (those of Andromeda VI, XI, XVII, XIX, XX, XXII, XXIII, XXV, XXVI, XXVIII and XXX [Casseopia II]), and from those presented by T12 (Andromeda I, III, V, VII, IX, X, XIII, XIV, XV and XVIII). For And XVI and XXI, we use newly derived values for the velocity dispersions of these objects that have been made from much larger samples of member stars $\left(\sigma_{v}=5.6 \pm 1.0\right.$ and $\sigma_{v}=5.4 \pm 0.9$ for XVI and XXI respectively, Collins et al. in prep). The velocity dispersion for Andromeda (And) II is taken from Ho et al. (2012), and that of And XXIX is taken from Tollerud et al. (2013). The velocity dispersion for And XII is unresolved, so we omit that from our study here. Owing to difficult observing conditions, the velocity dispersion of And XXIV is not well constrained, so we omit this value too. Finally, as And XXVII is likely a heavily disrupted system whose kinematics and structural properties are not well constrained (C13, Martin et al., in prep), we also remove this system from our analysis. This leaves us with a sample of 25 M31 dSph galaxies for which velocity dispersions have been reliably estimated. The structural properties are taken from McConnachie (2012) and Martin et al. (in prep., for the M31 dwarf galaxies that fall in the PAndAS footprint), updated based on the revised distances to the Andromeda dSphs presented in Conn et al. (2012).

\section{RESULTS}

\subsection{A universal mass profile?}

We focus our analysis here on the inclusion of the masses of the M31 dSphs into the universal density profile of Walker et al. (2009). In their work, the authors were spurred on by the earlier results of Strigari et al. (2008) that showed that all of the MW dSphs for which kinematic data were available were consistent with having the same mass contained within a radius of $300 \mathrm{pc}$ (roughly $1 \times 10^{7} \mathrm{M}_{\odot}$ ) despite spanning 6 decades in luminosity. Strigari et al. (2008) used this result to argue that it was possible that all dSphs inhabited a universal dark matter halo, where the density as a function of radius was identical, irrespective of the number of stars the halo hosted. However, Wolf et al. (2010) demonstrated that extrapolations to both larger and smaller radii than the true half light radius are extremely uncertain in cases where the velocity anisotropy is unknown, and this is true for all the Local Group dSphs. For objects with $r_{\text {half }}<<300$ pc one has to extrapolate to radii inhabited by no tracers, where tidal stripping may have removed the outer dark matter envelope (Peñarrubia et al. 2008b). That means that for some galaxies extrapolating out to $300 \mathrm{pc}$ could over-estimate the enclosed mass by several orders of magnitude. In the interest of trying to measure a more meaningful mass for these objects to determine whether dSphs truly resided within a universal halo, Walker et al. (2009) measured the velocity disper- sion, and hence mass, within the half-light radius of the MW dSphs. Then, by treating each velocity dispersion measurement from MW dSphs as a measurement of the velocity dispersion at a given radius (the half-light radius of the dSph in question) within a single dark matter halo, they could map out the velocity dispersion profile for this singular halo. In particular, they tested the cosmologically motivated Navarro Frenk White (NFW, Navarro et al. 1997) density profile:

$r_{\text {half }} \sigma_{v}^{2}=\frac{2 \eta R_{S} V_{\max }^{2}}{5} \times\left[\frac{\ln \left(1+r_{\text {half }} / R_{S}\right)-\frac{r_{\text {half }} / R_{S}}{1+r_{\text {half }} / R_{S}}}{\ln (1+\eta)-\frac{\eta}{1+\eta}}\right]$,

where $V_{\max }$ is the maximum circular velocity of the halo, $R_{S}$ is the scale radius of the halo and $\eta=2.16$. They also used a cored density profile where:

$$
\begin{array}{r}
r_{\text {half }} \sigma_{v}^{2}=\frac{2 \eta R_{S} V_{\max }^{2}}{5(\ln [1+\eta])+\frac{2}{1+\eta}-\frac{1}{2(1+\eta)^{2}}-\frac{3}{2}} \times \\
{\left[\ln \left(1+r_{\text {half }} / R_{S}\right)+\frac{2}{1+r_{\text {half }} / R_{S}}-\frac{1}{2\left(1+r_{\text {half }} / R_{S}\right)^{2}}-\frac{3}{2}\right],}
\end{array}
$$

with $\eta=4.42, \alpha=1$ and $\gamma=0$. The results of this study showed that the MW dSphs were consistent with having formed with a universal mass profile, although the authors noted that there was significant scatter about this relation, a factor of 2 greater than expected from the observational uncertainties alone. Later that same year, a revised study of the mass of the Hercules dSph (Adén et al. 2009), which provided a better treatment of the contaminating foreground population, determined a much lower value for the velocity dispersion of this object $\left(3.72 \pm 0.91 \mathrm{~km} \mathrm{~s}^{-1}\right.$ vs. $5.1 \pm 0.9 \mathrm{~km} \mathrm{~s}^{-1}$ from Simon \& Geha 2007). With their revised value, they showed that the mass of Hercules was not consistent with the universal mass profile. As Hercules is likely significantly affected by tides (Adén et al. 2009, Martin \& Jin 2010), this is perhaps not unexpected. Similarly, an analysis of the Böotes I dSph by Koposov et al. (2011), who used multi-epoch observations taken with the VLT and implemented an enhanced data reduction approach to measure extremely precise radial velocities, measured a velocity dispersion of $\sigma_{v}=4.6_{-0.6}^{+0.8} \mathrm{~km} \mathrm{~s}^{-1}$, significantly lower than that of $\sim 6.5 \mathrm{~km} \mathrm{~s}^{-1}$ reported in previous studies. This also renders the Böotes I dSph inconsistent with the universal mass profile.

In Walker et al. (2009), velocity dispersions for only 2 M31 dSphs (And II and IX) were available. We therefore fit NFW and cored density profiles (equations 1 and 2 ) to the velocity dispersions of the entire $\mathrm{dSph}$ population with $L>2 \times 10^{4} \mathrm{~L}_{\odot}$ (ensuring we probe the same luminosity regime in both the MW and M31), to see how well these populations can be fit with a single density profile. Both profiles have two free parameters of interest to fit, the circular velocity of the halo, $V_{\max }$ and the scale radius $R_{S}$. To constrain these values, we use a maximum likelihood fitting routine to determine the most probable values for these parameters by maximising the likelihood function, $\mathcal{L}$, defined as: 

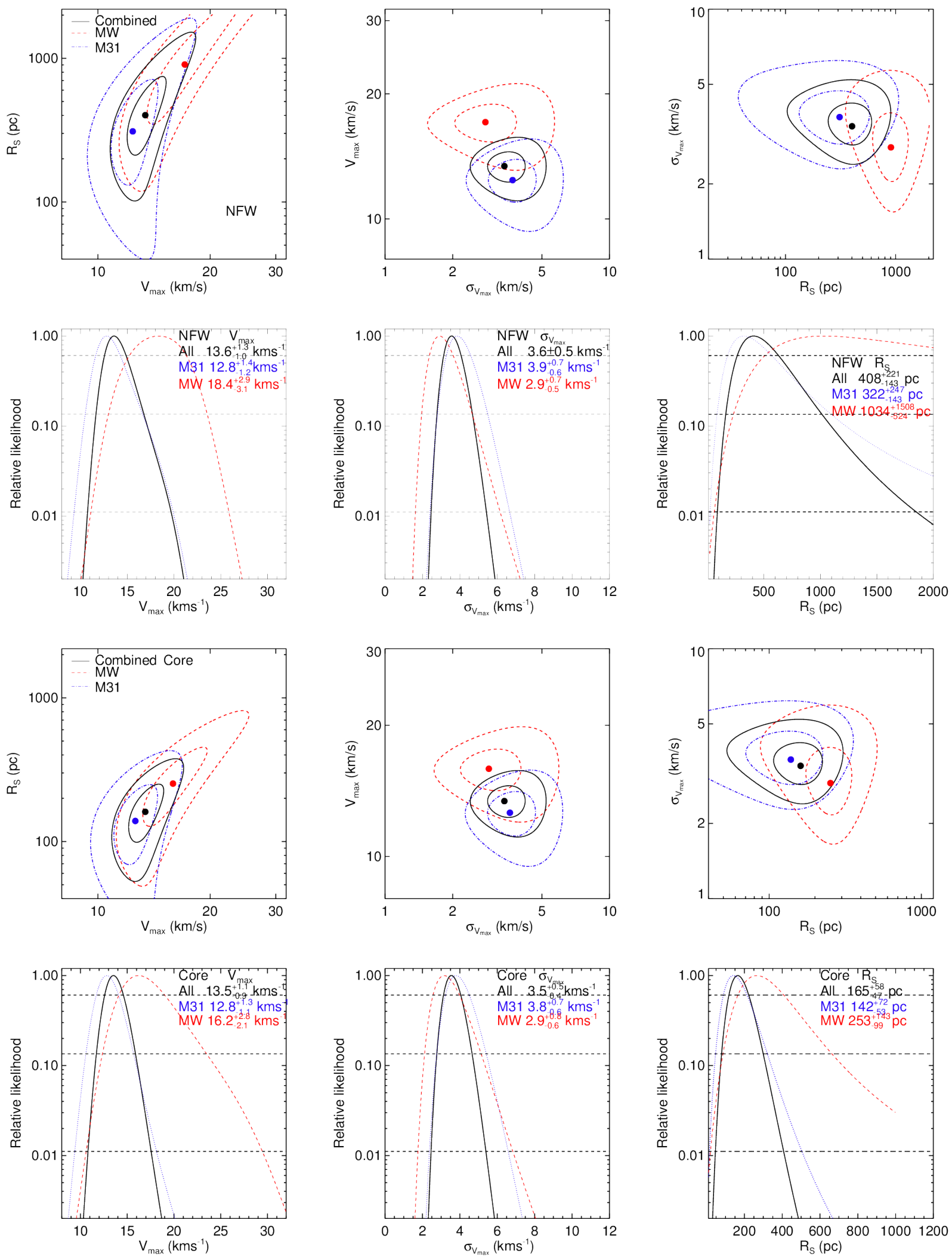

FIG. 2.- Top row: 2 dimensional likelihood contours for the three free parameters $\left(V_{\max }, \sigma_{V_{\max }}\right.$ and $\left.R_{S}\right)$ in the NFW mass profile fits to the MW dSphs (red dashed contours), M31 dSphs (blue dot-dashed contours) and all Local Group dSphs (solid black contours). The contours represent the 1 and $2 \sigma$ (i.e. $68 \%$ and 95\%) confidence intervals for these values. Second row: The resulting 1 dimensional marginalized likelihoods for $V_{\max }, \sigma_{V_{\max }}$ and $R_{S}$ (from left to right) for the MW, M31 and full sample. Horizontal dashed lines represent the 1,2 and $3 \sigma$ (i.e. $68 \%, 95 \%$ and $99.7 \%$ ) confidence intervals, derived assuming a Gaussian probability distribution. The best fit $\sigma_{V_{m}}$ and $R_{S}$ agree quite well between the MW and M31 case, however the values of $V_{\max }$ for the MW and M31 are marginally inconsistent, at the level of $1 \sigma$, with the M31 dSphs favoring fits with lower central masses. Third and fourth rows: As above six panels, but for cored density profile fits. Again, the best fit values of $V_{\max }$ for the MW and M31 are discrepant at the level of $1 \sigma$. 


$$
\begin{array}{r}
\mathcal{L}\left(\left\{r_{h, i}, \sigma_{v, i}, \delta_{\sigma_{v}, i}\right\} \mid V_{\max }, R_{S}\right)=\prod_{i=0}^{N} \frac{1}{\sqrt{2 \pi \delta_{\sigma_{v}, i}^{2}}} \\
\exp \left[-\frac{\left(\sigma_{\text {profile }}-\sigma_{v, i}\right)^{2}}{2 \delta_{\sigma_{v}, i}^{2}}\right]
\end{array}
$$

where $\sigma_{\text {profile }}$ is the velocity dispersion as predicted by equations 1 and 2 for a dSph with half-light radius $r_{h, i}$; $\sigma_{v, i}$ is the measured velocity dispersion of the $i^{\text {th }} \mathrm{dSph}$ and $\delta_{\sigma_{v}, i}$ is the uncertainty on the measured dispersion. We include only the uncertainty in $\sigma_{v}$ in our method, neglecting that of the half-light radius, as the velocity dispersion parameter has a greater impact on the mass profile, as it is proportional to the square of $\sigma_{v}$, depending only linearly on $r_{\text {half }}$.

We show the results of this fit in Fig. 1. The red triangles represent MW dSphs brighter than $L=2 \times 10^{4} \mathrm{~L}_{\odot}$, while open triangles represent those fainter than this cut. The blue circles are the M31 dSphs. The magenta dot-dashed line shows our best fit NFW profile to the whole population (with $V_{\max }=14.7 \pm 0.5 \mathrm{~km} \mathrm{~s}^{-1}$ and $R_{S}=876 \pm 284 \mathrm{pc}$ ), whilst the cyan dashed line is the best fit core profile (with $V_{\max }=14.0 \pm 0.4 \mathrm{~km} \mathrm{~s}^{-1}$ and $\left.R_{S}=242 \pm 124 \mathrm{pc}\right)$ where the best fit $V_{\max }\left(R_{S}\right)$ parameter is determined by marginalizing the $2 \mathrm{D}$ maximum likelihood contours over $R_{S}\left(V_{\max }\right)$. In all cases, the quoted uncertainties are derived by assuming that the likelihood functions have a Gaussian-like distribution, allowing us to project the marginalized maximum likelihood contours to the value at which $2 \ln (\mathcal{L})$ has decreased by the square of the confidence interval of interest, which in this case is the $1 \sigma$ (i.e. $68 \%$ ) confidence interval. Clearly, neither of these mass profiles is a good fit for many of the Local Group dSphs. This is statistically demonstrated by the reduced $\chi^{2}$ values for these fits $\left(\chi^{2}=4.1\right.$ for both profiles). Of the 39 objects, 24 are outliers at $>1 \sigma$, with $\sim 1 / 5$ of the population being outliers at the $3 \sigma$ level.

\subsection{Scatter about an average mass profile}

From the above analysis, it is clear that the scatter about the best fitting profiles is significant, and well beyond what we can hope to explain with measurement uncertainties. But do we really expect that all low luminosity galaxies should reside in dark matter halos with identical density profiles? The dark matter subhalos produced in, for example, the Aquarius simulations (Springel et al. 2008) demonstrate a range of possible values of $V_{\max }$ and $R_{s}$ for these objects. Further, work by e.g. Zolotov et al. (2012); Brooks \& Zolotov (2012) and Vera-Ciro et al. (2013) demonstrate that the infall time, host mass and presence of baryons can all effect the dark matter structures of subhalos. As such, scatter in density profiles is completely expected, and differences between the satellite populations of the Milky Way and Andromeda might also be seen that could tell us about the evolutionary histories of the two systems.

To investigate this, we introduce a mass-scatter term, $\sigma_{V_{\max }}$, into our maximum likelihood fitting algorithm (equation 3) replacing $\delta_{\sigma_{v}, i}$ with $\delta_{\text {tot, } i}$ which is the combination of the measured uncertainty in the velocity dispersion measurements and the mass-scatter term, such that $\delta_{\text {tot }, i}=\sqrt{\delta_{\sigma_{v}, i}^{2}+\sigma_{V_{\max }}^{2}}$.

If the mass profiles of the Andromeda and Milky Way dSphs are truly similar within their inner regions, our algorithm should find best fit values of $V_{\max }, R_{S}$ and $\sigma_{V_{\max }}$ that are broadly consistent when fitting the two populations separately and as a whole. In Figure 2 we show the likelihood contours for these parameters. In the top three panels we overlay the $1 \sigma$ and $2 \sigma$ confidence interval contours (again, defined as the region of parameter space where $2 \ln (\mathcal{L})$ decreases by the square of the confidence interval in question) for the NFW fits to the Milky Way (red dashed), Andromeda (blue dot-dashed) and the full sample (black solid) for our 3 free parameters (marginalized over the 3rd parameter not displayed in each 2D subplot), with solid points representing the best fit values in each case. In the second row of subplots, we show the one dimensional marginalised relative likelihood functions for $V_{\max }, R_{S}$ and $\sigma_{V_{\max }}$ for each of these fits. The lower 6 panels show the same, but for the cored fits. In the NFW case, while the best fit values for $R_{S}$ in each case seem dramatically different for the MW and M31 at first glance with $R_{S}=1034_{-524}^{+1508} \mathrm{pc}$ and $R_{S}=322_{-143}^{+247} \mathrm{pc}$ respectively, their uncertainties are such that they agree within $1 \sigma$. The amount of scatter in mass at a given radius is also very similar, with $\sigma_{V_{\max }}=$ $2.9_{-0.5}^{+0.7} \mathrm{~km} \mathrm{~s}^{-1}$ and $\sigma_{V_{\max }}=3.9_{-0.6}^{+0.7} \mathrm{~km} \mathrm{~s}^{-1}$ respectively for the MW and M31. The preferred values for $V_{\max }$ $\left(V_{\max }=18.4_{-3.1}^{+2.9} \mathrm{~km} \mathrm{~s}^{-1}\right.$ and $V_{\max }=12.8_{-1.2}^{+1.4} \mathrm{~km} \mathrm{~s}^{-1}$ respectively), however, are marginally less consistent, with M31 preferring a lower value of $V_{\max }$ (and hence, lower masses) than the MW system. For the core profiles, we get a similar result, with the values for $R_{S}$ $\left(R_{S}=253_{-99}^{+143} \mathrm{pc}\right.$ and $\left.R_{S}=142_{-53}^{+72} \mathrm{pc}\right)$, and $\sigma_{V_{\max }}$ $\left(\sigma_{V_{\max }}=2.9_{-0.6}^{+0.8} \mathrm{~km} \mathrm{~s}^{-1}\right.$ and $\left.\sigma_{V_{\max }}=3.8_{-0.6}^{+0.7} \mathrm{~km} \mathrm{~s}^{-1}\right)$ for the MW and M31 agreeing within $1 \sigma$, and marginally inconsistent values of $V_{\max }\left(V_{\max }=16.2_{-2.1}^{+2.8} \mathrm{~km} \mathrm{~s}^{-1}\right.$ and $V_{\max }=12.8_{-1.1}^{+1.3} \mathrm{~km} \mathrm{~s}^{-1}$ ).

In the top two panels of Fig. 3, we overplot the best fit relations from this analysis in the $r_{\text {half }}-\sigma_{v}$ plane. In the left panel, we show the best fit NFW profile for the MW (red line) and M31 (blue line) dSphs, with the best fit core profiles in the right panel. The shaded regions represent the scatter we derived convolved with the $1 \sigma$ uncertainties for $V_{\max }$ and $\sigma_{V_{\max }}$. Both the MW NFW and Core profiles provide an excellent fit to all the dSphs barring the Hercules dSph. However, as it is likely to be highly tidally disturbed (Adén et al. 2009, Martin \& Jin 2010), this is not too surprising. For the M31 fits, we see 3 systems that are outliers at the $\sim 2-3 \sigma$ level: And VI, VII and XXV. And XXV has previously been identified as an unusually low mass system in C13, so this inconsistency is perhaps not unexpected. However, And VI and VII are thought to represent fairly typical satellites, with velocity dispersions similar to their MW counterpart Fornax, which has a comparable half-light radius to these two objects.

The differences in the preferred $V_{\max }$ has a striking visual effect on the resulting best fit profiles for the MW and M31. While in both the NFW and Core case, the relations for MW and M31 populations track each other well at the smaller radius (lower mass) end (albeit with 

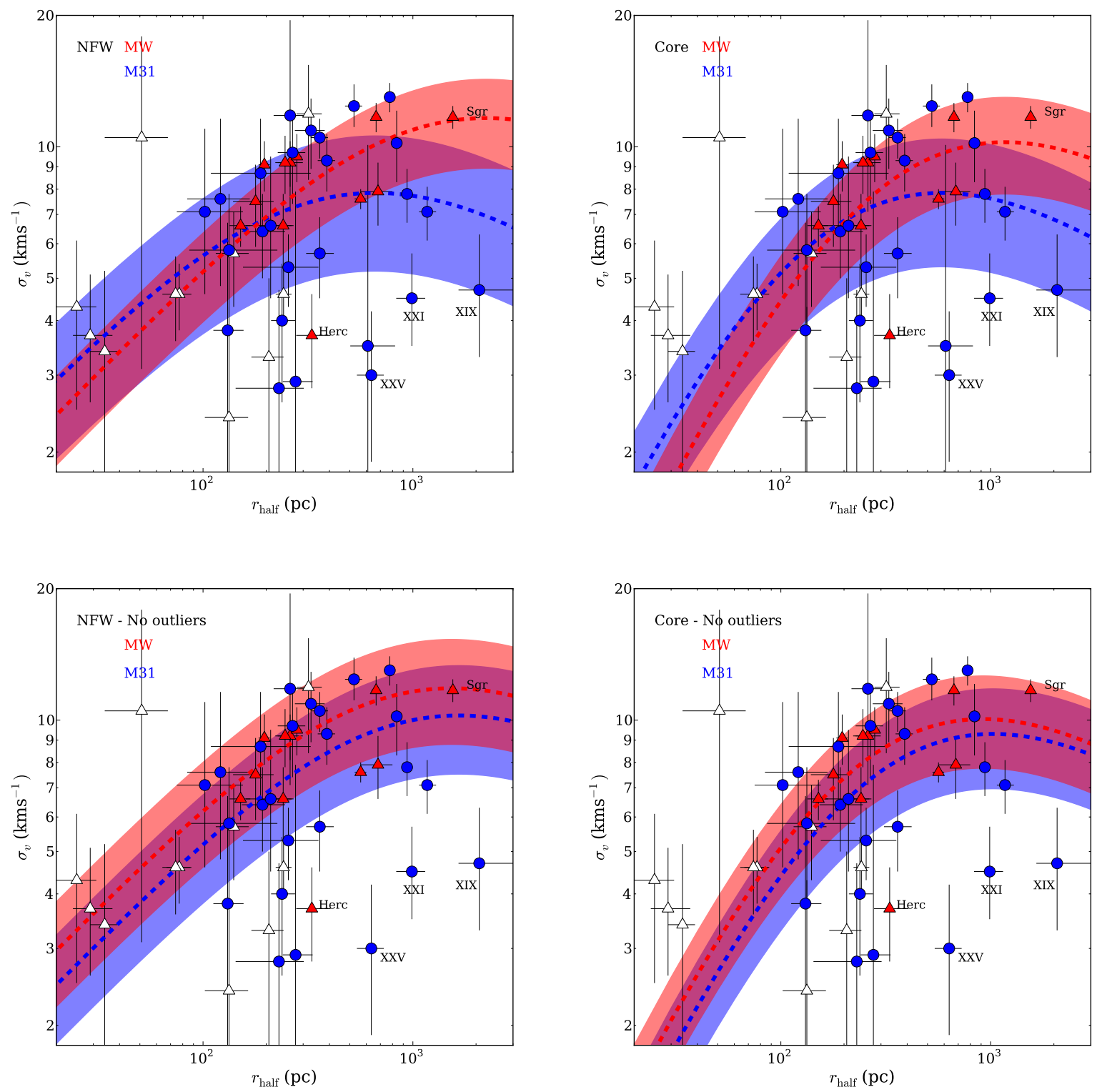

FIG. 3.- Top left: $\sigma_{v}$ vs. $r_{\text {half }}$ for all MW (red triangles) and M31 (blue circles) dSph galaxies. Overlaid are the best fit NFW profiles for the MW (red shaded region) and M31 (blue shaded region). The dashed lines represent the average fit while the shaded regions indicate the parameter space allowed by the introduction of the scatter term, $\sigma_{V_{\max }}$, convolved with the uncertainties in the fit parameters. At large $r_{\text {half }}$ (higher mass) the profiles of these populations begin to diverge, with the M31 fit turning over at $r_{\text {half }} \sim 600$ pc while the MW profile continues to rise. Top right: As top left, but with the best fit cored density profiles overlaid. Again, the M31 profile is seen to turn over before that of the MW profile. Bottom left and right: As top panels, but now the best fit profiles for NFW (left) and core (right) are determined after excluding And XIX, XXI and XXV. The removal of these objects results in best fit cored mass profile parameters that agree extremely well for MW and M31 dSphs.

greater scatter in M31), at larger radii there appears to be a divergence between the two systems. Both the NFW and core profiles for M31 begin to turnover at $\sim 600 \mathrm{pc}$, while the MW profile continues to rise (turning over at $\sim 1200 \mathrm{pc}$ in the cored case). This turnover radius is interesting, as there are only $3 \mathrm{MW}$ dSphs with half-light radii $\gtrsim 600 \mathrm{pc}$, one of which is the tidally disrupting Sagittarius (Sgr) and thus is excluded from our fits, the other two being Fornax and Sextans. In M31, there are 7 galaxies (And I, II, VII, XIX, XXI, XXIII and XXV), 3 of which (And XIX, XXI and XXV) are curiously very low mass for their size. In $\mathrm{C} 13$, they were measured to be $3 \sigma$ outliers to the best-fit mass profiles of Walker et al. (2009) and, as can be seen in Fig 3. they are significant outliers to the best fit MW relation $(2,2.5$ and $3 \sigma$ respectively). In addition, despite having very similar half-light radii, Sgr $\left(r_{\text {half }}=1550 \pm 50 \mathrm{pc}\right)$ and And XIX $\left(r_{\text {half }}=2072_{-422}^{+1092} \mathrm{pc}\right)$ have very different velocity dispersions $\left(\sigma_{v}=11.7 \pm 0.7 \mathrm{~km} \mathrm{~s}^{-1}\right.$ and $\left.\sigma_{v}=4.7_{-1.4}^{+1.6} \mathrm{~km} \mathrm{~s}^{-1}\right)$.

To see if it is these outliers driving the differences between the MW and M31 relations, we repeat the same fits performed above, but without all systems that were found to lie at or greater than $3 \sigma$ from the best fit pro- 

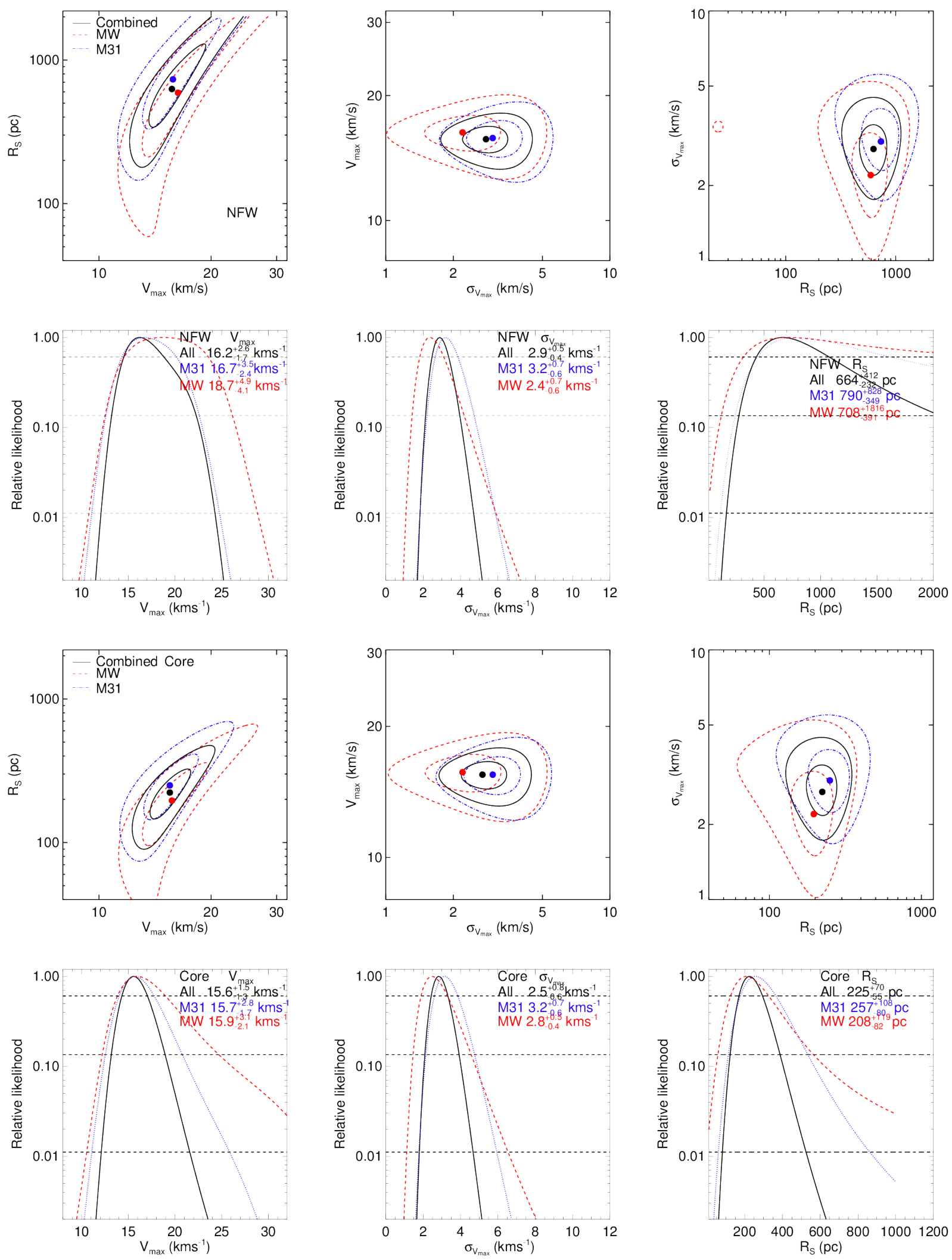

FIG. 4.- As Fig. 2, but with three significant low mass outliers, And XIX, XXI and XXV, omitted from the fits. The removal of these objects results in best fit NFW mass profile parameters that agree extremely well for MW and M31 dSphs. 
files of Walker et al. (2009). This included the three M31 outliers, and additionally the MW dSphs, Hercules and CVn I, which are also outliers to the Walker et al. (2009) relation. In Fig. 4 we show the same contours as in Fig. 2 for NFW and core profile fits, only this time with these outliers omitted. The exclusion of Hercules and CVn I from the MW fits has only a slight effect on these profiles, but removing the low mass M31 outliers is more substantial. The agreement between $R_{s}, V_{\max }$ and $\sigma_{V_{\max }}$ is significantly better (as shown in Table 1). In the lower two panels of Fig. 3, we show these best fit profiles in the $r_{\text {half }}-\sigma_{v}$ plane. Barring the 5 excluded dSphs and the M33 satellite, And XXII (Chapman et al. 2012), all the Local Group objects have velocity dispersions that are well described by the M31 and MW relations. The best fit profiles to the whole Local Group system are shown in Fig. 5. and have preferred values for the NFW (core) parameters of $R_{S}=664_{-232}^{+412} \mathrm{pc}\left(R_{S}=225_{-55}^{+70} \mathrm{pc}\right)$, $V_{\max }=16.2_{-1.7}^{+2.6} \mathrm{~km} \mathrm{~s}^{-1}\left(V_{\max }=15.6_{-1.3}^{+1.5} \mathrm{~km} \mathrm{~s}^{-1}\right)$ and $\sigma_{V_{\max }}=2.9_{-0.4}^{+0.5} \mathrm{~km} \mathrm{~s}^{-1}\left(\sigma_{V_{\max }}=2.8_{-0.4}^{+0.5} \mathrm{~km} \mathrm{~s}^{-1}\right)$. Therefore, whilst dSph galaxies do not live within dark matter halos with identical density profiles, the vast majority do inhabit statistically similar halos with a well defined mass range at any given radius.

In Fig. 5 we show the mass within the half-light radii of the dSphs (calculated using the Walker et al. 2009 mass estimator, where $M_{\text {half }}=580 r_{\text {half }} \sigma_{v}^{2}$, tabulated in Table 2) with the best fit NFW and Core relations when excluding the outliers. At all radii, the total scatter is less than half a magnitude in mass. For example, at $r_{\text {half }}=10 \mathrm{pc}, 100 \mathrm{pc}$ and $1000 \mathrm{pc}$, the average masses from the cored profile are $M_{\text {half }}=2.3 \times 10^{4} \mathrm{M}_{\odot}, 1.1 \times$ $10^{6} \mathrm{M}_{\odot}$ and $5.4 \times 10^{7} \mathrm{M}_{\odot}$, and the scatter (i.e. half the distance outlined by the shaded band) is $M_{\text {scatter }}=1.2 \times$ $10^{4} \mathrm{M}_{\odot}, 0.6 \times 10^{6} \mathrm{M}_{\odot}$ and $2.5 \times 10^{7} \mathrm{M}_{\odot}$, which is $\sim 50 \%$ of the average mass in each case. The numbers for the best fit NFW profile are almost identical.

Our decision to exclude And XIX, XXI and XXV was based on their designation as significant $(>3 \sigma)$ low mass outliers in $\mathrm{C} 13$, and to the derived MW profile. There are several other potentially low mass systems that were identified in C13 (namely And XXII) and T12 (And XIV, And XV and XXII also), which we did not exclude, simply because their associated uncertainties place them much closer to the regime of expected mass from the MW system. If they too were shown to be truly low mass with subsequent observations, this would imply that the M31 dwarf spheroidal systems do have greater scatter towards lower masses in their mass profiles compared with the MW.

\subsection{Comparing the observational scatter to simulations}

Briefly, we compare the best fit values of $V_{\max }$ and the scatter in this term with recent cosmological and semi-analytical models to deduce whether the values we statistically obtain for the Local Group dSphs compare favorably with our theoretical understanding of galaxy formation and evolution.

If we naively compare to dark-matter only simulations, such as the subhalos in the Aquarius simulations (Springel et al. 2008) of $6 \mathrm{MW}$-mass dark matter halos, we find that our measured values of $V_{\max }$ are lower than would be expected. The same discrepancy was pointed out by Boylan-Kolchin et al. (2012). While the MW dSphs have $12 \lesssim V_{\max } \lesssim 25 \mathrm{~km} \mathrm{~s}^{-1}$, they found at least 10 subhalos in each Aquarius host with $V_{\max }>25 \mathrm{~km} \mathrm{~s}^{-1}$. This discrepancy is referred to as the 'Too Big To Fail' problem, and would seem to persist when including M31 dSphs.

If we instead compare with models where baryons are taken into account, do we still see such inconsistencies? In Rashkov et al. (2012), dark matter subhalos from the high resolution Via Lactea II simulations are populated with baryons at the time of infall into their host halo by dynamically tagging dark matter particles as stars. These systems are then traced until $z=0$, where their final properties are compared to observations. These simulations are able to reproduce many observed properties of MW dSphs (such as velocity dispersions, sizes, metalicities, number count etc.), and that the present day values of $V_{\max }$ for the 10 most luminous subhalos are more compatible with observations, having $10<V_{\max }<40 \mathrm{~km} \mathrm{~s}^{-1}$ $\left(\sim 50 \%\right.$ of which are less than $\left.20 \mathrm{kms}^{-1}\right)$. Our average $V_{\text {max }}$ plus scatter term gives a statistical range for the $V_{\max }$ of the Local Group dSphs of $\sim 12-22 \mathrm{~km} \mathrm{~s}^{-1}$. So while the bulk of their sample is consistent, there remain too many high mass dSph satellites to be fully consistent. We can also compare our calculated values of $R_{S}$ with those of the Rashkov et al. (2012) simulations. The range of $R_{\max }$ (which is the radius at which the circular velocity of the halo is at a maximum, i.e., equal to $\left.V_{\max }\right)$ for their 10 most luminous subhalos ranges from $\sim 790-5400$ pc (assuming an NFW profile). According to Peñarrubia et al. (2008a), $R_{\max } \sim 2 R_{S}$, so this corresponds to scale radii for the Rashkov et al. (2012) halos of $395 \mathrm{pc} \lesssim R_{s} \lesssim 2700 \mathrm{pc}$ which is consistent with the scale radius of $R_{S}=664_{-232}^{+412} \mathrm{pc}$ that we find for our combined NFW profile (with outliers excluded), suggesting that these subhalos are similarly dense to the Local Group dSphs.

Bovill \& Ricotti (2011a) model satellites within the Local Volume from reionization until today, tracing the merger histories and tidal interactions of these objects as they merge to form more massive galaxies. As with the Rashkov et al. (2012) study, they are able to reproduce many of the observed properties of MW and M31 dSphs. For satellites with similar luminosities to those we fit in this work (i.e., $L \gtrsim 10^{4} \mathrm{~L}_{\odot}$ ) they measure $10 \lesssim V_{\max } \lesssim 30 \mathrm{~km} \mathrm{~s}^{-1}$ which is, again, largely consistent with the range of $V_{\max }$ we find. In this instance, the Bovill \& Ricotti (2011a) model produces more bright, massive satellites than we see in the Local Group. They discuss this in Bovill \& Ricotti (2011b) as the "missing bright satellite" problem. However, for the systems with comparable luminosities, there is significant overlap in their masses.

From these comparisons, we are content that the best fit profiles to the MW, M31 and total Local Group dSph we have derived are not hugely at odds with predictions from simulations. Some tension remains at the higher end of the subhalo mass range, as the simulations we compare with identify at least a few subhalos with greater $V_{\max }$ than are compatible with observations.

\section{THE MASS-TO-LIGHT RATIOS AND CIRCULAR VELOCITIES OF LOCAL GROUP DSPHS}


TABLE 1

Best fit parameters from mass profile fits to MW and M31 DSph DATA Using NFW and CORED PRofiles.

\begin{tabular}{|c|c|c|c|c|c|c|c|c|c|}
\hline \multirow[t]{2}{*}{ Model } & \multicolumn{3}{|c|}{ Full } & \multicolumn{3}{|c|}{ M31 } & \multicolumn{3}{|c|}{ MW } \\
\hline & $\begin{array}{c}V_{\max } \\
\left(\mathrm{km} \mathrm{s}^{-1}\right)\end{array}$ & $\begin{array}{l}R_{S} \\
(\mathrm{pc})\end{array}$ & $\begin{array}{c}\sigma_{V_{\max }} \\
\left(\mathrm{km} \mathrm{s}^{-1}\right)\end{array}$ & $\begin{array}{c}V_{\max } \\
\left(\mathrm{km} \mathrm{s}^{-1}\right)\end{array}$ & $\begin{array}{l}R_{S} \\
(\mathrm{pc})\end{array}$ & $\begin{array}{c}\sigma_{V_{\max }} \\
\left(\mathrm{km} \mathrm{s}^{-1}\right)\end{array}$ & $\begin{array}{c}V_{\max } \\
\left(\mathrm{km} \mathrm{s}^{-1}\right)\end{array}$ & $\begin{array}{l}R_{S} \\
(\mathrm{pc})\end{array}$ & $\begin{array}{c}\sigma_{V_{\max }} \\
\left(\mathrm{km} \mathrm{s}^{-1}\right)\end{array}$ \\
\hline NFW & $13.6_{-1.0}^{+1.3}$ & $408_{-143}^{+221}$ & $3.6 \pm 0.5$ & $12.8_{-1.2}^{+1.4}$ & $322_{-143}^{+247}$ & $3.9_{-0.6}^{+0.7}$ & $18.4_{-3.1}^{+2.9}$ & $1034_{-524}^{+1508}$ & $2.9_{-0.5}^{+0.7}$ \\
\hline NFW (minus outliers) & $16.2_{-1.7}^{+\frac{1}{2} .0}$ & $664_{-232}^{+143}$ & $2.9_{-0.5}^{+0.5}$ & $16.7_{-2.4}^{+1.2}$ & $790_{-349}^{+143}$ & $3.2_{-0.6}^{+0.6}$ & $18.7_{-4.1}^{+4.1}$ & $708_{-391}^{+1824}$ & $2.4_{-0.5}^{+0.5}$ \\
\hline Core & $13.5_{-0.9}^{+1.1}$ & $165_{-47}^{+58}$ & $3.5_{-0.4}^{+0.5}$ & $12.8_{-1.1}^{+1.4}$ & $142_{-53}^{+72}$ & $3.8_{-0.6}^{+0.0}$ & $16.2_{-2.1}^{+2.1}$ & $253_{-99}^{+141}$ & $2.9_{-0.6}^{+0.8}$ \\
\hline Core (minus outliers) & $15.6_{-1.3}^{+1.9}$ & $225_{-55}^{+76}$ & $2.8_{-0.4}^{+0.4}$ & $15.7_{-1.7}^{+1.1}$ & $257_{-88}^{+108}$ & $3.2_{-0.6}^{+0.6}$ & $15.9_{-2.1}^{+3.1}$ & $208_{-82}^{+119}$ & $2.5_{-0.6}^{+0.6}$ \\
\hline
\end{tabular}
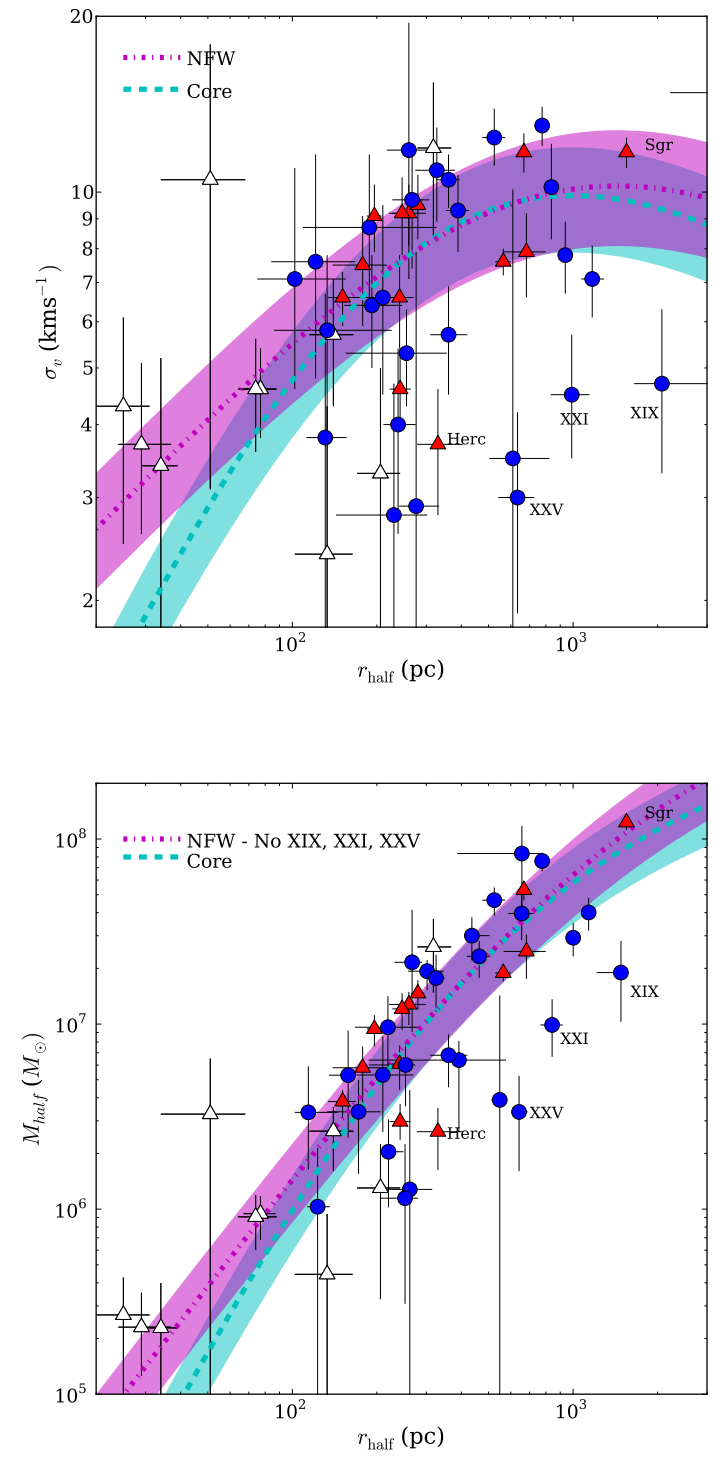

FIG. 5.- The best fit NFW (magenta) and core (cyan) relations to the whole Local Group population as seen in the $r_{\text {half }}-\sigma_{v}$ (top) and $r_{\text {half }}-M_{\text {half }}$ (bottom) planes. We see that the velocity dispersions and masses for all the dSphs, barring the excluded outliers and 3 further objects (discussed further in the text) agree with the fits to the whole Local Group population within their uncertainties.
TABLE 2

The masses, mass-to-Light Ratios, and $V_{\max }$ VAlues FOR LOCAL GROUP DSPHS AS DERIVED IN THIS WORK

\begin{tabular}{|c|c|c|c|}
\hline Name & $\begin{array}{c}M_{\text {half }} \\
\left(\times 10^{7} \mathrm{M}_{\odot}\right)\end{array}$ & $\begin{array}{l}{[M / L]_{\text {half }}} \\
\left(\mathrm{M}_{\odot} / \mathrm{L}_{\odot}\right)\end{array}$ & $\begin{array}{c}V_{c, 1 / 2} \\
\left(\mathrm{~km} \mathrm{~s}^{-1}\right)\end{array}$ \\
\hline AndI & $5.05 \pm 1.35$ & $22.4 \pm 8.5$ & $16.1 \pm 4.4$ \\
\hline AndII & $3.31 \pm 0.7$ & $27.6 \pm 10.8$ & $12.3 \pm 2.6$ \\
\hline AndIII & $1.95 \pm 0.46$ & $39.0 \pm 12.9$ & $14.7 \pm 3.7$ \\
\hline AndV & $2.30 \pm 0.40$ & $78.0 \pm 19.5$ & $16.6 \pm 3.3$ \\
\hline AndVI & $4.67 \pm 0.9$ & $27.5_{-6.85}^{+7.61}$ & $19.5_{-3.9}^{+4.2}$ \\
\hline AndVII & $7.61 \pm 0.9$ & $9.0 \pm 1.6$ & $20.5 \pm 2.7$ \\
\hline AndIX & $2.25 \pm 0.7$ & $302 \pm 132$ & $17.2 \pm 6.0$ \\
\hline AndX & $0.46 \pm 0.2$ & $61.2_{-49}^{+52}$ & $10.1_{-4.3}^{+5.1}$ \\
\hline AndXI & $0.41_{-0.2}^{+0.3}$ & $165.5_{-142}^{+196}$ & $12.0_{-8.1}^{+11.0}$ \\
\hline AndXIII & $0.26_{-0.16}^{+0.22}$ & $126.6_{-108}^{+153}$ & $9.2_{-6.1}^{+10.1}$ \\
\hline AndXIV & $0.42 \pm 0.2$ & $41.6 \pm 28.2$ & $\begin{array}{l}8.4 \pm 5.2 \\
8.4\end{array}$ \\
\hline AndXV & $0.22 \pm 0.11$ & $\begin{array}{c}9.0_{-7.0}^{+7.1} \\
\text {. }\end{array}$ & $6.3_{-3.3}^{+3.4}$ \\
\hline AndXVI & $0.24_{-0.06}^{+0.08}$ & $11.6_{-2.9}^{+3.9}$ & $8.8_{-2.7}^{+3.2}$ \\
\hline AndXVII & $0.13_{-0.19}^{+0.33}$ & $12.82_{-26.38}^{+44.79}$ & $4.5_{-4.5}^{+11.2}$ \\
\hline AndXVIII & $1.5 \pm 0.5$ & $44.8 \pm 27.1$ & $15.3 \pm 6.1$ \\
\hline AndXIX & $2.7_{-1.2}^{+1.9}$ & $118.0_{-85.2}^{+124.2}$ & $7.4_{-3.8}^{+6.6}$ \\
\hline AndXX & $0.30_{-0.17}^{+0.28}$ & $213.0_{-171.0}^{+282.2}$ & $11.2_{-7.0}^{+12.0}$ \\
\hline AndXXI & $1.2_{-0.4}^{+0.5}$ & $29.8_{-16.5}^{+18.7^{\circ}}$ & $7.1_{-2.7}^{+3.1}$ \\
\hline AndXXII & $0.10_{-0.08}^{+0.11}$ & $69.7_{-82.4}^{+102.2}$ & $4.4_{-3.9}^{+4.7}$ \\
\hline AndXXIII & $3.4_{-0.7}^{+0.80}$ & $68.4_{-46.1}^{+46.4}$ & $\begin{array}{r}-3.9 \\
11.2_{-2.6}^{+2.8}\end{array}$ \\
\hline AndXXV & $0.33_{-0.18}^{+0.19}$ & $10.2_{-9.5}^{+10.0}$ & $4.7_{-2.6}^{+2.9}$ \\
\hline AndXXVI & $0.83_{-0.46}^{+0.72}$ & $279.8_{-277.6}^{+383}$ & $13.7_{-9.6}^{+15.6}$ \\
\hline AndXXVIII & $0.53_{-0.27}^{+0.36}$ & $50.5_{-39.1}^{+51.0^{-6}}$ & $\begin{array}{r}-9.6 \\
10.4_{-5.8}^{+7.7}\end{array}$ \\
\hline AndXXIX & $0.68_{-0.22}^{+0.23}$ & $67.8_{-37.5}^{+38.1}$ & $9.0_{-3.2}^{+3.4}$ \\
\hline AndXXX & $2.1_{-1.2}^{+2.0}$ & $300.0_{-302.3}^{+433.1}$ & $18.6_{-11.4}^{+17.7}$ \\
\hline Scl & $1.3 \pm 0.3$ & $\begin{array}{l}18.2 \pm 9.8 \\
\end{array}$ & $14.5 \pm 3.9$ \\
\hline LeoT & $0.58 \pm 0.22$ & $196.9 \pm 120.0$ & $11.8 \pm 5.1$ \\
\hline UMaI & $2.6_{-1.1}^{+1.2}$ & $3731_{-2524}^{+2577}$ & $18.8_{-8.5}^{+8.9}$ \\
\hline LeoIV & $0.13 \pm 0.10$ & $299.1 \pm 54.2$ & $5.2 \pm 4.0$ \\
\hline Com & $0.09 \pm 0.03$ & $510.8 p m 309.0$ & $7.3 \pm 2.2$ \\
\hline CVnII & $0.09 \pm 0.03$ & $229.9_{-152}^{+158}$ & $7.3_{-2.6}^{+3.0}$ \\
\hline LeoI & $1.2 \pm 0.3$ & $7.1 \pm 3.3$ & $14.5 \pm 3.5$ \\
\hline LeoII & $0.38 \pm 0.07$ & $12.9 \pm 5.2$ & $10.4 \pm 2.2$ \\
\hline Car & $0.6 \pm 0.2$ & $50.7 \pm 28.9$ & $10.4 \pm 3.0$ \\
\hline UMi & $1.5 \pm 0.3$ & $146.6 \pm 76.5$ & $15.0 \pm 2.9$ \\
\hline Dra & $0.94 \pm 0.18$ & $69.7 \pm 22.0$ & $14.4 \pm 3.0$ \\
\hline For & $5.3 \pm 0.6$ & $7.6 \pm 2.5$ & $18.5 \pm 2.4$ \\
\hline Sex & $2.5 \pm 0.71$ & $120.4 \pm 74.4$ & $12.5 \pm 4.2$ \\
\hline Boo & $0.30_{-0.06}^{+0.08}$ & $198.0_{-69.1}^{+83.4}$ & $7.3_{-1.6}^{+2.0}$ \\
\hline CVnI & $\begin{array}{l}1.9 \pm 0.2 \\
-0.06\end{array}$ & $\begin{array}{l}164.3 \pm 31.2 \\
-69.1\end{array}$ & $12.0 \pm 1.4$ \\
\hline Herc & $0.26_{-0.10}^{+0.11}$ & $145.6_{-89.7}^{+95.8}$ & $5.8_{-2.4}^{+2.8}$ \\
\hline LeoV & $0.04 \pm 0.05$ & $197.5 \pm 339.1$ & $3.8 \pm 4.4$ \\
\hline Wil1 & $0.03 \pm 0.02$ & $536.2 \pm 613.8$ & $6.8 \pm 4.6$ \\
\hline UmaII & $0.26 \pm 0.10$ & $1319.1 \pm 961$ & $9.0 \pm 3.9$ \\
\hline Seg & $0.02 \pm 0.01$ & $1374.7_{-1234.16}^{+1453.47}$ & $5.8_{-2.8}^{+3.9}$ \\
\hline Seg2 & $0.02 \pm 0.02$ & $536.4 \pm 588.7$ & $4.1_{-4.1}^{+0.9}$ \\
\hline
\end{tabular}


In Fig. 6, we plot the masses contained within the halflight radii $\left(M_{\text {half }}\right)$ of all the Local Group dSphs as a function of their luminosity within the half-light radius $\left(L_{\text {half }}\right)$, where the points are colour coded as in previous figures. The values themselves can be found in Table 2 . Additionally we overplot lines of constant mass-to-light ratio (with $[M / L]_{\text {half }}=1,10,100$ and 1000). It can be seen that the majority of these objects (including two of our outliers, And XIX and XXI, labeled in plot) have $[M / L]_{\text {half }} \gtrsim 10$, indicating that their dynamical masses are much higher than can feasibly be explained by the mass of their baryons alone (although see recent work in predicting the velocity dispersions and mass-to-light ratios of M31 dSphs using MOND, without dark matter by McGaugh \& Milgrom 2013). This is typically ascribed to the presence of dark matter halos in these objects, whose mass dominates that of their baryons. The green shaded region in this plot represents the parameter space in this framework typically inhabited by globular cluster systems of the MW (Rejkuba et al. 2007), whose masses can be explained by their stellar content alone, without invoking dark matter.

Interestingly, we see a few objects on this plot whose mass-to-light ratios are consistent (within $1 \sigma$ uncertainties) with those of the Galactic globular clusters, suggesting that they possess little or no dark matter. In a couple of cases, the very large uncertainties on current measurements mean that this overlap is not significant, and will likely disappear with future observations. But there are two objects, And XV and XXV, that are particular noteworthy. The masses of And XV and XXV are derived from sample sizes of $\sim 30$ stars. The potential implication of this is that these galaxies contain very little dark matter, which would be quite unexpected for objects of their sizes and luminosities.

In Fig. 7] we plot the circular velocities measured within the half-light radius (a good proxy for the central mass of these objects) of the Local Group dSphs as a function of their half-light radii. We derive $V_{c, 1 / 2}$ from the measured masses within the half-light radius using the relationship between circular velocity and mass: $V_{c, 1 / 2}=\sqrt{\frac{G M_{\text {half }}}{r_{\text {half }}}}$. These values are tabulated in Table 2. The shaded regions overplotted represent the circular velocity profiles of Aquarius subhalos (taken from Boylan-Kolchin et al. 2012 ) and are labeled with their maximum circular velocity in each case. Subhalos with maximum circular velocities below $10 \mathrm{~km} \mathrm{~s}^{-1}$ (i.e. below the cyan profile in Fig. 7 ) are proposed to be too low mass to form luminous galaxies, as their star formation is highly suppressed due to inefficient gas cooling, causing them to remain essentially dark $\left(V_{\text {max,limit }} \sim 10 \mathrm{kms}^{-1}\right.$, Koposov et al. 2009). The red and blue curves in the figure are representative of the $\sim 10$ most-massive subhalos seen in DM only simulations where we would naively expect the most luminous dwarf galaxies in the Local Group to reside. When including the M31 dSphs, we see that there are now a number of systems that may be consistent with living in such massive halos. However, many of these systems are the less luminous objects $\left(-6>M_{V}>-8\right)$, where our measurement uncertainties are large. For the brighter M31 dSphs whose velocity dispersions (and hence, masses) are well resolved, there are only 2 objects (And VI and And VII) that may inhabit halos with maximum circular veloci-

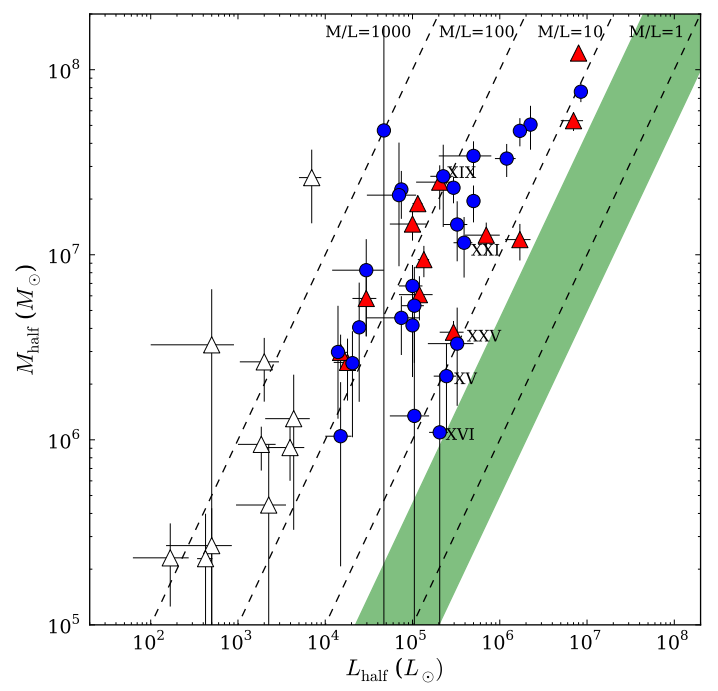

FIG. 6.- Mass within the half-light radius $\left(M_{\text {half }}\right)$ as a function of luminosity within the half-light radius $\left(L_{\text {half }}\right)$ for Local Group dSphs. Points are colour coded as in previous figures. The dashed lines represent mass-to-light ratios of $[M / L]_{\text {half }}=1,10,100$ and $1000 \mathrm{M}_{\odot} / \mathrm{L}_{\odot}$. The green shaded region indicates the parameter space typically inhabited by simple stellar systems (i.e., those without dark matter). It is interesting to note that there are a number of M31 objects, namely And XV and XXV, that are consistent with this regime.

ties of $24 \mathrm{~km} \mathrm{~s}^{-1}$ or greater. As such, the TBTF problem would seem to be present in Andromeda as well as the Milky Way.

The previously discussed outliers from C13 and T12 (Hercules, And XIV, XV, XVI, XIX, XXI and XXV) are all labeled in Fig. 7, as is the MW dSph, Boötes I (Boo I). These objects again stand out as they fall tentatively shy of the pre-reionization star formation threshold. If their halos have always been so low mass, they should never have been able to form stars. Given the large uncertainties, all but 4 of these outliers are (just) consistent with this lower limit,and thus, not of great concern. But And XXII and XXV in M31, and Herc and Boo I in the $\mathrm{MW}$, all fall below this threshold, even when taking their uncertainties into account. This implies that, in order for us to observe these systems now, their masses must have been higher in the past, and have been reduced by some physical process during their evolution. We discuss this further in $\S 5$.

Combined, these low mass-to-light ratios and lower than predicted masses, highlight the ongoing tensions between observations and theory. It is clear that, if the predictions from the $\Lambda$ CDM paradigm are to be reconciled with our observations, we must investigate avenues that can lower the masses of dark matter subhalos over the course of their cosmic evolution. In the next section, we discuss numerous possibilities for this that have been put forth recently, and comment on their ability to reproduce our findings within the Local Group.

\section{EXPLAINING THE MASSES OF THE LOCAL GROUP} DSPHS - OBSERVATIONS VS. THEORY

From the analysis in $\S 3$ and $\S 4$ we still see some discrepancies between the masses of subhalos in simu- 


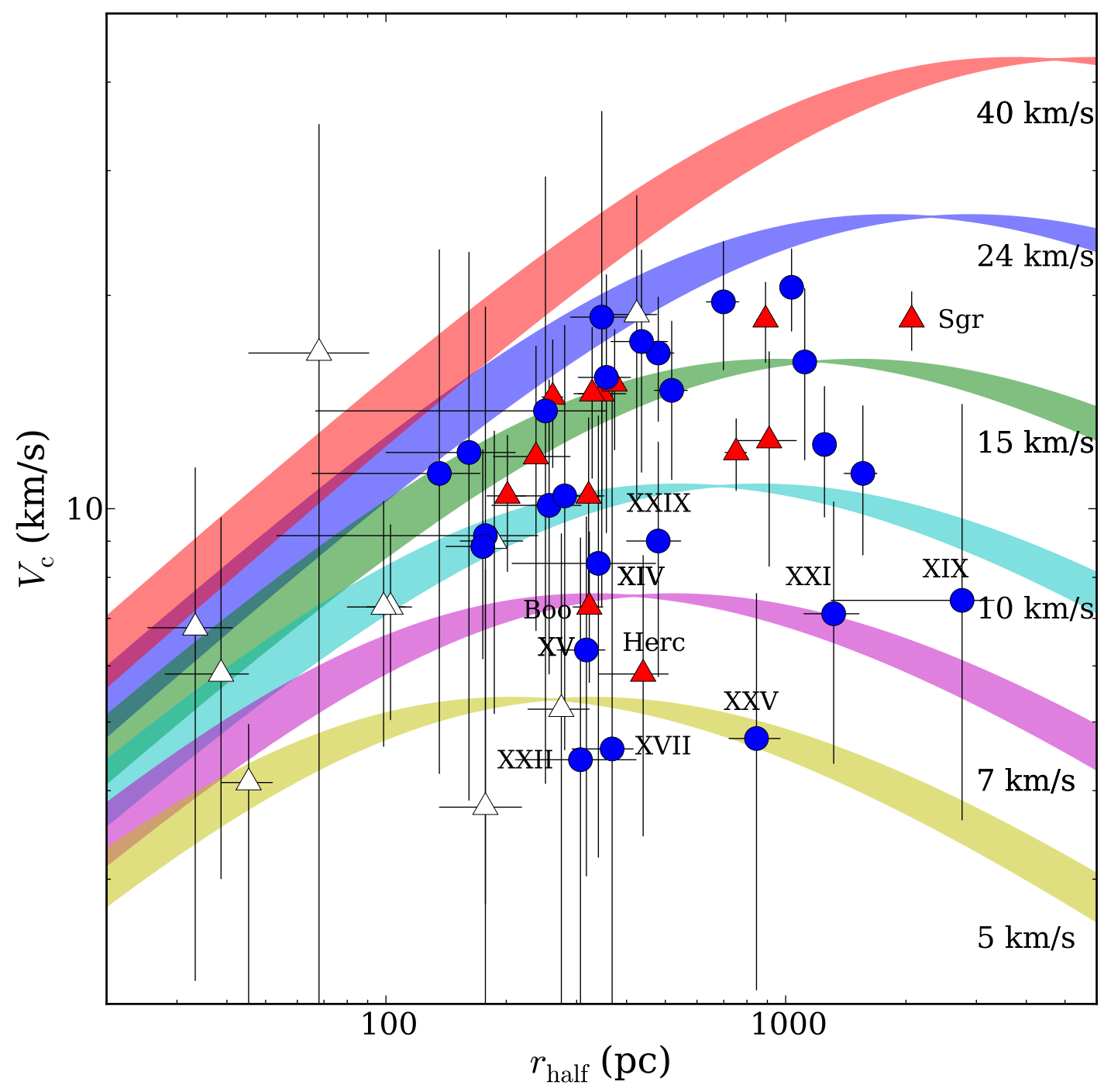

FIG. 7.- Circular velocities within the half-light radius $\left(V_{c, 1 / 2}\right)$ for Local Group dSphs, as derived from their velocity dispersions. The shaded lines are circular velocity profiles of subhalos from the Aquarius simulations (taken from Boylan-Kolchin et al. 2012), and are labeled with their maximum circular velocities. Subhalos with maximum circular velocities below $10 \mathrm{~km} \mathrm{~s}^{-1}$ (i.e. profiles below the cyan shaded curve) are not thought to be massive enough to efficiently cool their hydrogen and form stars. A number of dSphs (particularly Herc and And XXV) have circular velocities that see them preferentially residing in halos that fall below this low mass cut-off for star formation.

lations, and the masses we infer from observations of the subhalos within the Local Group. At face value, it seems that we expect to observe luminous satellites around MW mass halos with higher central masses than we do. One explanation for this missing massive satellite problem could simply be that at these low halo masses $\left(M_{\text {halo }}<10^{10} \mathrm{M}_{\odot}\right)$ star formation becomes increasingly stochastic, so that the luminosity of a subhalo does not necessarily correlate with the mass of the subhalo (Kuhlen et al. 2012, 2013). Other solutions appeal to physical processes affecting the evolution of dwarf galaxies, and can be broadly assigned to three categories: The effect of tidal interactions with the host galaxy, the effect of stellar feedback on the mass profiles of galax- ies and the true mass of the host system. Finally, some have also appealed to the modification of the current cosmological paradigm, $\Lambda \mathrm{CDM}$, either via the properties of the dark matter itself (e.g. warm dark matter, Anderhalden et al. 2013 or self interacting dark matter, Rocha et al. 2013 Zavala et al. 2013), or via the modification of Newtonian gravity (i.e. MOND, McGaugh \& Milgrom 2013 ) to remove the need for dark matter altogether. In the subsequent sections, we discuss the physical processes that might be responsible for lowering the central masses of the whole Local Group population, and why this effect might be more pronounced in some objects (i.e. Boo I, Herc, And XIX, XXI and And XXV) than others. 


\subsection{Tides}

The notion that the lower central masses observed in the outlying dSphs is the result of some physical process that has moved them away from a more typical mass is appealing, and the possibility has been briefly discussed in other works, particularly Collins et al. (2011) and $\mathrm{C} 13$. There, the authors point out that dSphs that are more extended at a given luminosity, such as the extreme outliers, And XIX, XXI and XXV, also tend to have lower central masses. One of the MW low mass outliers, the MW Herc object, is also already thought to be a tidally disrupting system, transitioning into a stellar stream (Martin \& Jin 2010).

Outside of the Local Group, a number of tidally disrupting dwarf galaxy systems have recently been observed whose half-light radii are also much more extended than would be expected from the $r_{\text {half }}-L$ relation (Brasseur et al. 2011), resulting in very low surface brightnesses. 'These include NCG 4449B $\left(M_{V}=-13.4\right.$ and $r_{\text {half }}=2.7 \mathrm{kpc}$, Rich et al. 2012, Martínez-Delgado et al. 2012), and the Hydra dwarf galaxy, HCC-087 $\left(M_{V}=-11.6\right.$ and $r_{\text {half }}=3.1 \mathrm{kpc}$, Koch et al. 2012). Their closest analog within the Local Group is And XIX $\left(M_{V}=-9.3, r_{\text {half }}=2.1_{-0.4}^{+1.0} \mathrm{kpc}\right.$, Martin et al. in prep.) making it an outlier to the Brasseur et al. (2011) relation, as well as falling below the mass expectation for a galaxy of its radial scale. Taking these examples into account, perhaps these properties (low surface brightness and/or low mass) are indicators of a system undergoing significant tidal interaction with its host.

Mass loss of subhalos from interactions with their hosts has also been studied in numerical models, both in a dark matter only context (e.g., Tormen et al. 1998; Klypin et al. 1999; Ghigna et al. 2000; Hayashi et al. 2003; Zentner \& Bullock 2003; Kravtsov et al. 2004b; Kazantzidis et al. 2004) and with the inclusion of baryons (e.g., Peñarrubia et al. 2010, D'Onghia et al. 2010; Zolotov et al. 2012; Brooks \& Zolotov 2012). In all cases, as these systems orbit their host, their dark matter is stripped, lowering their densities and masses at all radii. After the dark matter is removed, the stars reach a dynamic equilibrium with their lower density potential, causing a drop in the central mass. In simulations where baryonic physics are included, the mass losses from subhalos as a result of tidal interactions with a host are more pronounced than for the dark matter only case. Further, the size of the mass reduction increases with earlier infall times and more radial orbits. In Zolotov et al. (2012), they demonstrated that a subhalo accreted at $z>6 \mathrm{Gyr}$ in an SPH simulation would experience a greater reduction in its mass than is seen with a dark matter only set up. Similarly, the mass of subhalos on radial orbits in the SPH simulation also experience a more significant drop in mass than their dark matter only counterparts. In all cases, the presence of a massive baryonic disk in the host galaxy (such as those hosted by the Galaxy and M31) reduces the masses of the satellite population at a much greater rate than in the dark matter only case.

One could therefore argue that the outliers seen in this study, such as Hercules, And XIX, XXI and XXV, may have fallen in to their host galaxies earlier, and onto more radial orbits where they interact more significantly with their host, leading to a more pronounced mass loss. It is difficult to properly model the orbital properties of these objects, but recent work by Watkins et al. (2013) modelled the orbital properties of M31 dSphs by combining the timing argument with phase-space distribution functions. This work found no evidence to suggest that the M31 outliers are on very radial orbits, nor do they seem to have experienced particularly close passages with M31 itself, perhaps ruling out this option.

A prime example of a tidally disrupting $\mathrm{dSph}$ within the MW is the Sgr dSph. This object is currently undergoing violent tidal disruption, yet it has a velocity dispersion that is entirely consistent with the best fit NFW and cored mass profiles to both the MW alone and to the full Local Group, perhaps arguing against the mechanism we have outlined above. However, Sgr is currently near the pericenter of its orbit, only $\sim 20 \mathrm{kpc}$ from the Galactic center (Law \& Majewski 2010). The outliers we refer to are located further out $\left(D_{\text {host }}>70 \mathrm{kpc}\right.$ for all outliers, Martin \& Jin 2010; Koposov et al. 2011; Conn et al. 2013), and so we do not expect them to be currently experiencing significant tidal distortions, rather that their past interactions with their host have removed more mass from their centers than their more 'typical' counterparts.

In summary, numerical models have demonstrated that tidal mechanisms are able to lower the masses of dSphs, and could explain the lower than expected masses of the Local Group outliers, Herc, And XIV, XV, XVI, XIX, XXI and XXV if they have experienced more significant past interactions with their host.

\subsection{Feedback from star formation and supernova}

For many years, kinematic studies of low surface brightness galaxies have shown that the mass profiles of these objects are less centrally dense than expected. They are more compatible with flatter, cored halo functions, rather than the cuspier NFW profiles seen in simulations (e.g. Flores \& Primack 1994, de Blok \& Bosma 2002; de Blok et al. 2003; de Blok 2005). Many have argued that this is a result of bursty, energetic star formation and supernova (SN) within these galaxies. These processes drive mass out from the center of the halo, flattening the high density cusp into a lower density core, leading to a lower central mass than predicted by pure dark matter simulations (e.g. Navarro et al. 1996; Dekel \& Woo 2003; Read \& Gilmore 2005; Mashchenko et al. 2006; Pontzen \& Governato 2012, Governato et al. 2012; Macciò et al. 2012). Could the lower than expected central masses of the Local Group dSphs also be caused by feedback?

Zolotov et al. (2012) and Brooks \& Zolotov (2012) compared a dark matter only simulation with a smooth particle hydrodynamic ( $\mathrm{SPH}$ ) simulation of a MW type galaxy in a cosmological context to see whether the inclusion of baryons and feedback in the latter can produce satellite galaxies with lower central masses and densities. For galaxies with a stellar mass $M_{*}>10^{7} \mathrm{M}_{\odot}\left(M_{V} \lesssim-12\right)$ at the time of infall, feedback can reduce the central mass of dSph galaxies. Below this mass, the galaxies have an insufficient total mass to retain enough gas beyond reionization to continue with the significant, bursty star formation processes required to remove mass from their centers. The Local Group outliers discussed above have $M_{V} \gtrsim-10$, so unless they have been significantly 
tidally stripped by their hosts after falling in, i.e., experienced total (dark matter plus baryonic) mass losses of greater than 90\% (Peñarrubia et al. 2008b), feedback cannot explain their current masses. This is supported by the findings of Garrison-Kimmel et al. (2013), where they model the dynamical effect of SN feedback on the mass distribution of dark matter halos. To match the current observed central masses in MW dSph galaxies, one would need to deposit $100 \%$ of the energy resulting from 40, 000 SN directly to the dark matter halos, which is greater than the expected total number of SN to have ever occurred in the majority of these systems. The work of Peñarrubia et al. (2012) also support the findings of these works, namely that fainter dSphs should not be able to significantly lower their central masses via feedback, and if they were able, they would serve to exacerbate the missing satellite problem.

In Di Cintio et al. (2013), they also study the effect of feedback in subhalos on their mass profiles, using a suite of galaxies from the MaGICC project (Stinson et al. 2010, 2013). Their findings show that the mass of stars formed per halo mass is the most important factor for the shaping of the central mass profiles of galaxies. Objects with stellar-to-halo mass ratios of $M_{*} / M_{\text {halo }} \lesssim 0.01$ are not able to alter their dark matter distributions, but as this fraction increases, so does the ability to flatten their central mass profiles. They find that this process is maximally efficient in galaxies with $M_{*} \approx 10^{8.5} \mathrm{M}_{\odot}$, and below this, the central dark matter slope increases once more. As such, their findings are similar to those of Zolotov et al. (2012). This seeming consensus on the amount of baryons required to efficiently reshape the dark matter mass profile of a dwarf galaxies via feedback means that, in principle, based on their current luminosities only 4 of the dSphs discussed in this work (And II, And VII, Fornax, and Leo I, McConnachie 2012) have enough explosive energy at their disposal to reduce their central densities with feedback alone. For the remaining MW and M31 objects, another mechanism, such as tides, would need to be invoked to explain their low masses.

\subsection{Host mass}

From a plethora of works (e.g. Moore et al. 1999. Ghigna et al.|2000; Kravtsov et al.||2004a; Zentner et al. 2005: van den Bosch et al. 2005: Zheng et al. 2005: Giocoli et al. 2008; Springel et al. 2008), we know that the number of subhalos within a host halo, scales with the mass of the host halo itself. Therefore, when comparing the mass of halos we observe within the MW or Andromeda with those found in simulations, it is important that we select a simulated galaxy of the same mass. Unfortunately, in the case of both M31 and the MW, the total masses of these systems are actually quite uncertain, ranging from $\sim 0.7-2.7 \times 10^{12} \mathrm{M}_{\odot}$ for the Milky Way (e.g., Wilkinson \& Evans 1999: Xue et al. 2008; Li \& White 2008, Watkins et al. 2010; Piffl et al. 2013) and $\sim 0.8-2.2 \times 10^{12} \mathrm{M}_{\odot}$ (e.g., Evans \& Wilkinson 2000; Evans et al. 2000; Li \& White 2008, Guo et al. 2010; Watkins et al. 2010), making this difficult. From the point of view of abundance matching, a galaxy as luminous as the MW should be hosted by a halo with an even higher mass than these estimates $\left(\sim 3 \times 10^{12} \mathrm{M}_{\odot}\right.$, Behroozi et al. 2013), which could imply that our Galaxy is a significant outlier when compared with the bulk of the galaxies within the Uni- verse.

Vera-Ciro et al. (2013) investigated the effect of varying the mass of the host halo on both the number and dynamics of subhalos using the Aquarius simulations (Springel et al. 2008). They found that they were able to match both these quantities when using a simulated halo whose mass was consistent with the lower bound of observational constraints for the MW, $8 \times 10^{11} \mathrm{M}_{\odot}$. This immediately eliminates the TBTF problem, as the most massive simulated subhalos have $V_{\max } \lesssim 25 \mathrm{~km} \mathrm{~s}^{-1}$. Di Cintio et al. (2012) also find that they can match the number and dynamics of MW satellites using halos from the CLUES simulations with masses of $5-7 \times 10^{11} \mathrm{M}_{\odot}$ (Gottloeber et al. 2010, Di Cintio et al. 2012). Thus, if the virial mass of the MW is at the lower end of current observational estimates, the masses we measure for its subhalos would be much more inline with predictions from numerical simulations. However, it is worth noting that such a low mass for the MW would lessen the probability of hosting massive satellites like the LMC and $\mathrm{SMC}$, and may just replace the missing massive satellite problem with a found massive satellite problem (e.g. Boylan-Kolchin et al. 2011a; Busha et al. 2011). It is also in conflict with a recent estimate of the mass of the MW from Boylan-Kolchin et al. (2013) who use the Aquarius simulations to demonstrate that the $3 \mathrm{D}$ space motion of the Leo I dSph puts a lower limit of $\sim 1 \times 10^{12} \mathrm{M}_{\odot}$ on the mass of the MW at a confidence level of $95 \%$. Vera-Ciro et al. (2013) also find that the Andromeda satellites can be best matched if the host mass is $1.77 \times 10^{12} \mathrm{M}_{\odot}$, implying that the mass of M31 is roughly twice that of the MW. This value is compatible with the best estimate for the Andromeda mass when using the full dwarf galaxy satellite population as a tracer (L. Watkins, private communication). If Andromeda is more massive than the MW, it could explain the fact that M31 has more (in number terms) massive non-dSph satellites (NGC147, NGC 185, NGC 205, M32 and M33) than the MW (LMC and SMC). It may also explain the low mass outliers, particularly the statistically significant And XIX, XXI and $\mathrm{XXV}$. In particular, the very low value of $V_{c, 1 / 2}$ we derive for And XXV strongly indicates that it is currently residing in a dark matter subhalo with a maximum circular velocity below the mass limit expected for luminous galaxy formation, suggesting it has experienced some physical process that has lowered its mass significantly over the course of its evolution. A more massive host would imply that the dSphs (which are more susceptible to tidal disruption than the more massive satellites) within this system have experienced greater tidal forces over the course of their evolution, which could lower their masses below the SF threshold of $10-15 \mathrm{~km} \mathrm{~s}^{-1}$ (Peñarrubia et al. 2008b; Koposov et al. 2009).

Precisely pinning down the correct viral masses of the MW and M31 is clearly an important step towards better understanding the masses of the dwarf galaxies we observe within the Local Group in a cosmological context. Without precise mass estimates, it is difficult for us to quantify discrepancies with theoretical expectations, like those of the TBTF problem, and might help us to explain the differences between the masses of dSphs we see around M31 and the MW.

\section{CONCLUSIONS}


The relatively high dark-to-stellar mass ratios of dSph galaxies single them out as excellent probes of the behaviour of dark matter on the smallest of scales. Comparisons of the masses of the MW dSphs with expectations from cosmological simulations have revealed several discrepancies, most notably the issue of cuspy vs. cored central densities and a dearth of luminous high mass subhalos around the MW compared with dark matter only simulations (the 'too big to fail' problem). In this paper we have expanded these analyses by including the dSph satellites of M31 in the comparisons.

We revisit the notion that all $\mathrm{dSph}$ are embedded in dark matter halos that follow a universal density profile in their centers (Walker et al. 2009) by fitting NFW and cored mass profiles to the full sample of MW and M31 dSphs in $r_{\text {half }}-\sigma_{v}$ space. We find that no singular profile provides a good fit to the data, but that their masses are instead described by a range of halo profiles with a well defined scatter as a function of half light radius. We find that when comparing fits for solely MW dSphs to solely M31 dSphs, the latter prefer significantly lower masses for a given size than the former. We demonstrate that this offset is driven by 3 low mass outliers in M31, whose half-light radius place them in a region of parameter space with very few MW dSphs for comparison (And XIX, XXI, and XXV). Once these outliers are removed, we find that the two populations agree exceptionally well, following mass profiles with similar average values of $V_{\max }$ and $R_{S}$, and a scatter in mass that equates to $\sim 50 \%$ of the average mass at any specific radius.

We also derive the $V_{c, 1 / 2}$ values for each dSph directly from their velocity dispersions and find them to be in good agreement with our fitted ranges, with the exception of the 3 excluded outliers. Further inspection of these values, plus the mass-to-light ratios of the population reveal a number of interestingly low mass systems. The most significant of these are And XV, XIX, XXI and XXV from M31 and Herc and Boo I from the MW. In particular, the central mass of And XXV is so low that if it had always been this way, it would never have formed stars. And yet, now; it is clearly luminous. By comparing the properties of these objects with those of observed tidally disrupting dwarf galaxies, we postulate that tides are a candidate mechanism for lowering the masses of these objects, especially when combined with stellar feedback at early epochs that can reduce the central masses of these galaxies before they fall into their host.

When comparing the computed values of $V_{c, 1 / 2}$ from our observed sample with the circular velocity profiles of subhalos within simulations, we still see an offset between the most massive simulated subhalos, and the most massive dSphs in both the MW and M31, described as the TBTF problem. We argue that, as this problem was defined via comparisons with a dark matter only simulation of a MW type halo that neglects the effects on the mass profiles of dSphs from baryonic processes (such as feedback and tides), and may not be directly comparable to the MW and M31 (given the uncertainties on observational measurements of their masses), it is difficult to quantify how serious or significant this problem truly is. By running simulations with baryonic processes included, and precisely determining the masses of the MW and M31, we will better be able to assess whether there is truly a missing massive satellite problem. As such, the masses of Local Group dSphs should be thought of as a constraint for more complex simulations that include a wide range of physical processes that are not currently well understood.

\section{ACKNOWLEDGMENTS}

We would like to thank the anonymous referee for their helpful comments and suggestions, as well as a thorough discussion surrounding the 'Too Big To Fail' problem.

The research leading to these results has received funding from the European Research Council under the European Union's Seventh Framework Programme (FP 7) ERC Grant Agreement n. [321035].

R.I. gratefully acknowledges support from the Agence Nationale de la Recherche though the grant POMMME (ANR 09-BLAN-0228).

G.F.L. and N.B. gratefully acknowledge financial support for his ARC Future Fellowship (FT100100268) and through the award of an ARC Discovery Project (DP110100678).

A.K. thanks the Deutsche Forschungsgemeinschaft for funding from Emmy-Noether grant Ko 4161/1.

\section{REFERENCES}

Adén, D., Wilkinson, M. I., Read, J. I., Feltzing, S., Koch, A., Gilmore, G. F., Grebel, E. K., \& Lundström, I. 2009, ApJ, 706, L150

Amorisco, N. C. \& Evans, N. W. 2012, MNRAS, 419, 184

Anderhalden, D., Schneider, A., Macciò, A. V., Diemand, J., \& Bertone, G. 2013, JCAP, 3, 14

Behroozi, P. S., Wechsler, R. H., \& Conroy, C. 2013, ApJ, 770, 57

Bovill, M. S. \& Ricotti, M. 2011a, ApJ, 741, 17

-. 2011b, ApJ, 741, 18

Boylan-Kolchin, M., Besla, G., \& Hernquist, L. 2011a, MNRAS, 414, 1560

Boylan-Kolchin, M., Bullock, J. S., \& Kaplinghat, M. 2011b, MNRAS, 415, L40

-. 2012, MNRAS, 422, 1203

Boylan-Kolchin, M., Bullock, J. S., Sohn, S. T., Besla, G., \& van der Marel, R. P. 2013, ApJ, 768, 140

Brasseur, C. M., Martin, N. F., Macciò, A. V., Rix, H.-W., \& Kang, X. 2011, ApJ, 743, 179

Brooks, A. M. \& Zolotov, A. 2012, ArXiv e-prints

Busha, M. T., Wechsler, R. H., Behroozi, P. S., Gerke, B. F., Klypin, A. A., \& Primack, J. R. 2011, ApJ, 743, 117
Chapman, S. C., Widrow, L., Collins, M. L. M., Dubinski, J., Ibata, R. A., Rich, M., Ferguson, A. M. N., Irwin, M. J., Lewis, G. F., Martin, N., McConnachie, A., Penarrubia, J., \& Tanvir, N. 2012, ArXiv e-prints

Collins, M. L. M., Chapman, S. C., Irwin, M. J., Martin, N. F., Ibata, R. A., Zucker, D. B., Blain, A., Ferguson, A. M. N., Lewis, G. F., McConnachie, A. W., \& Peñarrubia, J. 2010, MNRAS, 407, 2411

Collins, M. L. M., Chapman, S. C., Rich, R. M., Ibata, R. A., Martin, N. F., Irwin, M. J., Bate, N. F., Lewis, G. F.,

Peñarrubia, J., Arimoto, N., Casey, C. M., Ferguson, A. M. N., Koch, A., McConnachie, A. W., \& Tanvir, N. 2013, ApJ, 768, 172

Collins, M. L. M., Chapman, S. C., Rich, R. M., Irwin, M. J., Peñarrubia, J., Ibata, R. A., Arimoto, N., Brooks, A. M. Ferguson, A. M. N., Lewis, G. F., McConnachie, A. W., \& Venn, K. 2011, MNRAS, 417, 1170

Conn, A. R., Ibata, R. A., Lewis, G. F., Parker, Q. A., Zucker, D. B., Martin, N. F., McConnachie, A. W., Irwin, M. J., Tanvir, N., Fardal, M. A., Ferguson, A. M. N., Chapman, S. C., \& Valls-Gabaud, D. 2012, ApJ, 758, 11 
Conn, A. R., Lewis, G. F., Ibata, R. A., Parker, Q. A., Zucker, D. B., McConnachie, A. W., Martin, N. F., Valls-Gabaud, D., Tanvir, N., Irwin, M. J., Ferguson, A. M. N., \& Chapman, S. C. 2013, ApJ, 766, 120

de Blok, W. J. G. 2005, ApJ, 634, 227

de Blok, W. J. G. \& Bosma, A. 2002, A\&A, 385, 816

de Blok, W. J. G., Bosma, A., \& McGaugh, S. 2003, MNRAS, 340,657

Dekel, A. \& Woo, J. 2003, MNRAS, 344, 1131

Di Cintio, A., Brook, C. B., Maccio, A. V., Stinson, G. S., Knebe, A., Dutton, A. A., \& Wadsley, J. 2013, ArXiv e-prints

Di Cintio, A., Knebe, A., Libeskind, N. I., Brook, C., Yepes, G., Gottloeber, S., \& Hoffman, Y. 2012, ArXiv e-prints

D'Onghia, E., Springel, V., Hernquist, L., \& Keres, D. 2010, ApJ, 709,1138

Evans, N. W. \& Wilkinson, M. I. 2000, MNRAS, 316, 929

Evans, N. W., Wilkinson, M. I., Guhathakurta, P., Grebel, E. K., \& Vogt, S. S. 2000, ApJ, 540, L9

Flores, R. A. \& Primack, J. R. 1994, ApJ, 427, L1

Garrison-Kimmel, S., Rocha, M., Boylan-Kolchin, M., Bullock, J., \& Lally, J. 2013, ArXiv e-prints

Ghigna, S., Moore, B., Governato, F., Lake, G., Quinn, T., \& Stadel, J. 2000, ApJ, 544, 616

Giocoli, C., Tormen, G., \& van den Bosch, F. C. 2008, MNRAS, 386,2135

Gottloeber, S., Hoffman, Y., \& Yepes, G. 2010, ArXiv e-prints

Governato, F., Zolotov, A., Pontzen, A., Christensen, C., Oh, S. H., Brooks, A. M., Quinn, T., Shen, S., \& Wadsley, J. 2012, MNRAS, 422, 1231

Guo, Q., White, S., Li, C., \& Boylan-Kolchin, M. 2010, MNRAS, 404,1111

Hayashi, E., Navarro, J. F., Taylor, J. E., Stadel, J., \& Quinn, T. 2003, ApJ, 584, 541

Ho, N., Geha, M., Munoz, R. R., Guhathakurta, P., Kalirai, J., Gilbert, K. M., Tollerud, E., Bullock, J., Beaton, R. L., \& Majewski, S. R. 2012, ApJ, 758, 124

Jardel, J. R. \& Gebhardt, K. 2012, ApJ, 746, 89

Kalirai, J. S., Beaton, R. L., Geha, M. C., Gilbert, K. M., Guhathakurta, P., Kirby, E. N., Majewski, S. R., Ostheimer, J. C., Patterson, R. J., \& Wolf, J. 2010, ApJ, 711, 671

Kazantzidis, S., Mayer, L., Mastropietro, C., Diemand, J., Stadel, J., \& Moore, B. 2004, ApJ, 608, 663

Kirby, E. N., Boylan-Kolchin, M., Cohen, J. G., Geha, M., Bullock, J. S., \& Kaplinghat, M. 2013, ApJ, 770, 16

Klypin, A., Kravtsov, A. V., Valenzuela, O., \& Prada, F. 1999, ApJ, 522, 82

Koch, A., Burkert, A., Rich, R. M., Collins, M. L. M., Black, C. S., Hilker, M., \& Benson, A. J. 2012, ApJ, 755, L13

Komatsu, E., Smith, K. M., Dunkley, J., Bennett, C. L., Gold, B., Hinshaw, G., Jarosik, N., Larson, D., Nolta, M. R., Page, L., Spergel, D. N., Halpern, M., Hill, R. S., Kogut, A., Limon, M., Meyer, S. S., Odegard, N., Tucker, G. S., Weiland, J. L., Wollack, E., \& Wright, E. L. 2011, ApJS, 192, 18

Koposov, S. E., Gilmore, G., Walker, M. G., Belokurov, V., Wyn Evans, N., Fellhauer, M., Gieren, W., Geisler, D., Monaco, L., Norris, J. E., Okamoto, S., Penarrubia, J., Wilkinson, M., Wyse, R. F. G., \& Zucker, D. B. 2011, ArXiv e-prints, 1105.4102

Koposov, S. E., Yoo, J., Rix, H.-W., Weinberg, D. H., Macciò, A. V., \& Escudé, J. M. 2009, ApJ, 696, 2179

Kravtsov, A. V., Berlind, A. A., Wechsler, R. H., Klypin, A. A., Gottlöber, S., Allgood, B., \& Primack, J. R. 2004a, ApJ, 609 35

Kravtsov, A. V., Gnedin, O. Y., \& Klypin, A. A. 2004b, ApJ, 609,482

Kuhlen, M., Krumholz, M. R., Madau, P., Smith, B. D., \& Wise, J. 2012, ApJ, 749, 36

Kuhlen, M., Madau, P., \& Krumholz, M. 2013, ArXiv e-prints

Law, D. R. \& Majewski, S. R. 2010, ApJ, 714, 229

Li, Y. \& White, S. D. M. 2008, MNRAS, 384, 1459

Macciò, A. V., Stinson, G., Brook, C. B., Wadsley, J., Couchman, H. M. P., Shen, S., Gibson, B. K., \& Quinn, T. 2012, ApJ, 744, L9

Martin, N. F. \& Jin, S. 2010, ApJ, 721, 1333
Martínez-Delgado, D., Romanowsky, A. J., Gabany, R. J., Annibali, F., Arnold, J. A., Fliri, J., Zibetti, S., van der Marel, R. P., Rix, H.-W., Chonis, T. S., Carballo-Bello, J. A., Aloisi, A., Macciò, A. V., Gallego-Laborda, J., Brodie, J. P., \& Merrifield, M. R. 2012, ApJ, 748, L24

Mashchenko, S., Couchman, H. M. P., \& Wadsley, J. 2006, Nature, 442, 539

Mateo, M. L. 1998, ARA\&A, 36, 435

McConnachie, A. W. 2012, AJ, 144, 4

McGaugh, S. \& Milgrom, M. 2013, ApJ, 766, 22

Moore, B., Ghigna, S., Governato, F., Lake, G., Quinn, T., Stadel, J., \& Tozzi, P. 1999, ApJ, 524, L19

Navarro, J. F., Eke, V. R., \& Frenk, C. S. 1996, MNRAS, 283, L72

Navarro, J. F., Frenk, C. S., \& White, S. D. M. 1997, ApJ, 490, 493

Peñarrubia, J., Benson, A. J., Walker, M. G., Gilmore, G., McConnachie, A. W., \& Mayer, L. 2010, MNRAS, 406, 1290

Peñarrubia, J., McConnachie, A. W., \& Navarro, J. F. 2008a, ApJ, 672, 904

Peñarrubia, J., Navarro, J. F., \& McConnachie, A. W. 2008b, ApJ, 673, 226

Peñarrubia, J., Pontzen, A., Walker, M. G., \& Koposov, S. E. 2012, ApJ, 759, L42

Piffl, T., Scannapieco, C., Binney, J., Steinmetz, M., Scholz, R.-D., Williams, M. E. K., de Jong, R. S., Kordopatis, G., Matijevic, G., Bienayme, O., Bland-Hawthorn, J., Boeche, C., Freeman, K., Gibson, B., Gilmore, G., Grebel, E. K., Helmi, A., Munari, U., Navarro, J. F., Parker, Q., Reid, W. A., Seabroke, G., Watson, F., Wyse, R. F. G., \& Zwitter, T. 2013, ArXiv e-prints

Planck Collaboration, Ade, P. A. R., Aghanim, N., Armitage-Caplan, C., Arnaud, M., Ashdown, M. Atrio-Barandela, F., Aumont, J., Baccigalupi, C., Banday, A. J., \& et al. 2013a, ArXiv e-prints

-. 2013b, ArXiv e-prints

Pontzen, A. \& Governato, F. 2012, MNRAS, 421, 3464

Rashkov, V., Madau, P., Kuhlen, M., \& Diemand, J. 2012, ApJ, 745,142

Read, J. I. \& Gilmore, G. 2005, MNRAS, 356, 107

Read, J. I., Wilkinson, M. I., Evans, N. W., Gilmore, G., \& Kleyna, J. T. 2006, MNRAS, 367, 387

Rejkuba, M., Dubath, P., Minniti, D., \& Meylan, G. 2007, A\&A, 469,147

Rich, R. M., Collins, M. L. M., Black, C. M., Longstaff, F. A., Koch, A., Benson, A., \& Reitzel, D. B. 2012, Nature, 482, 192

Rocha, M., Peter, A. H. G., Bullock, J. S., Kaplinghat, M., Garrison-Kimmel, S., Oñorbe, J., \& Moustakas, L. A. 2013 , MNRAS, 430, 81

Simon, J. D. \& Geha, M. 2007, ApJ, 670, 313

Springel, V., Wang, J., Vogelsberger, M., Ludlow, A., Jenkins, A., Helmi, A., Navarro, J. F., Frenk, C. S., \& White, S. D. M. 2008, MNRAS, 391, 1685

Stinson, G. S., Bailin, J., Couchman, H., Wadsley, J., Shen, S. Nickerson, S., Brook, C., \& Quinn, T. 2010, MNRAS, 408, 812

Stinson, G. S., Brook, C., Macciò, A. V., Wadsley, J., Quinn, T. R., \& Couchman, H. M. P. 2013, MNRAS, 428, 129

Strigari, L. E., Bullock, J. S., Kaplinghat, M., Simon, J. D., Geha, M., Willman, B., \& Walker, M. G. 2008, Nature, 454, 1096

Tollerud, E. J., Beaton, R. L., Geha, M. C., Bullock, J. S., Guhathakurta, P., Kalirai, J. S., Majewski, S. R., Kirby, E. N., Gilbert, K. M., Yniguez, B., Patterson, R. J., Ostheimer, J. C., Cooke, J., Dorman, C. E., Choudhury, A., \& Cooper, M. C. 2012, ApJ, 752, 45

Tollerud, E. J., Bullock, J. S., Strigari, L. E., \& Willman, B. 2008, ApJ, 688, 277

Tollerud, E. J., Geha, M. C., Vargas, L. C., \& Bullock, J. S. 2013, ArXiv e-prints

Tormen, G., Diaferio, A., \& Syer, D. 1998, MNRAS, 299, 728 van den Bosch, F. C., Tormen, G., \& Giocoli, C. 2005, MNRAS, 359,1029

Vera-Ciro, C. A., Helmi, A., Starkenburg, E., \& Breddels, M. A. 2013, MNRAS, 428, 1696

Walker, M. G., Mateo, M., Olszewski, E. W., Peñarrubia, J.,

Wyn Evans, N., \& Gilmore, G. 2009, ApJ, 704, 1274

Walker, M. G. \& Peñarrubia, J. 2011, ApJ, 742, 20

Walsh, S. M., Willman, B., \& Jerjen, H. 2009, AJ, 137, 450 
Watkins, L. L., Evans, N. W., \& An, J. H. 2010, MNRAS, 406, 264

Watkins, L. L., Evans, N. W., \& van de Ven, G. 2013, MNRAS, 430, 971

Wilkinson, M. I. \& Evans, N. W. 1999, MNRAS, 310, 645

Wolf, J., Martinez, G. D., Bullock, J. S., Kaplinghat, M., Geha, M., Muñoz, R. R., Simon, J. D., \& Avedo, F. F. 2010, MNRAS, 406, 1220

Xue, X. X., Rix, H. W., Zhao, G., Re Fiorentin, P., Naab, T. Steinmetz, M., van den Bosch, F. C., Beers, T. C., Lee, Y. S., Bell, E. F., Rockosi, C., Yanny, B., Newberg, H., Wilhelm, R., Kang, X., Smith, M. C., \& Schneider, D. P. 2008, ApJ, 684, 1143
Zavala, J., Vogelsberger, M., \& Walker, M. G. 2013, MNRAS, 431, L20

Zentner, A. R., Berlind, A. A., Bullock, J. S., Kravtsov, A. V., \& Wechsler, R. H. 2005, ApJ, 624, 505

Zentner, A. R. \& Bullock, J. S. 2003, ApJ, 598, 49

Zheng, Z., Berlind, A. A., Weinberg, D. H., Benson, A. J., Baugh, C. M., Cole, S., Davé, R., Frenk, C. S., Katz, N., \& Lacey, C. G. 2005, ApJ, 633, 791

Zolotov, A., Brooks, A. M., Willman, B., Governato, F., Pontzen, A., Christensen, C., Dekel, A., Quinn, T., Shen, S., \& Wadsley, J. 2012, ArXiv e-prints 